\title{
REPRESENTATIONS OF QUADRATIC JORDAN ALGEBRAS
}

\author{
BY \\ KEVIN MCCRIMMON
}

\begin{abstract}
Although representations do not play as much of a role in the theory of Jordan algebras as they do in the associative or Lie theories, they are important in considering Wedderburn splitting theorems and other applications. In this paper we develop a representation theory for quadratic Jordan algebras over an arbitrary ring of scalars, generalizing the usual theory for linear Jordan algebras over a field of characteristic $\neq 2$. We define multiplication algebras and representations, characterize these abstractly as quadratic specializations, and relate them to bimodules. We obtain first and second cohomology groups with the usual properties. We define a universal object for quadratic specializations and show it is finite dimensional for a finite-dimensional algebra. The most important examples of quadratic representations, those obtained from commuting linear representations, are discussed and examples are given of new "pathological" representations which arise only in characteristic 2.
\end{abstract}

\section{The Unital Case}

Throughout this paper $\Phi$ will denote an arbitrary unital, commutative, associative ring of scalars and $\mathfrak{X}$ an arbitrary unital $\Phi$-module. A map $q: \mathfrak{X} \rightarrow \mathfrak{Y}$ is quadratic (with respect to $\Phi$ ) if it is homogeneous of degree $2, q(\alpha x)=\alpha^{2} q(x)$ for $\alpha \in \Phi$, and if $q(x, y)=q(x+y)-q(x)-q(y)$ is bilinear in $x$ and $y$. A unital quadratic Jordan algebra over $\Phi$ is a triple $\mathfrak{I}=(\mathfrak{X}, U, 1)$ where $\mathfrak{X}$ is a $\Phi$-module, $x \rightarrow U(x)$ $=U_{x}$ is a quadratic map from $\mathfrak{X}$ to $\operatorname{Hom}_{\Phi}(\mathfrak{X}, \mathfrak{X})$, and $1 \in \mathfrak{X}$ is a unit element such that the identities

(UQJ I) $U_{1}=I$,

(UQJ II) $U_{U(x) y}=U_{x} U_{y} U_{x}$,

(UQJ III) $U_{U(x) y, x}=U_{x} V_{y, x}=V_{x, y} U_{x}$

hold strictly (in the sense that they remain valid under any extension of the ring of scalars-this is equivalent to the intrinsic condition that the linearizations of UQJ I-III hold over the original ring of scalars), where

(1) $V_{x, y} z=\{x y z\}=U_{x, z} y=\left\{U_{x+z}-U_{x}-U_{z}\right\} y$.

We have a squaring operation $x^{2}=U_{x} 1$, and by linearization we obtain a symmetric bilinear product

$$
V_{x} y=x \circ y=(x+y)^{2}-x^{2}-y^{2}
$$

Received by the editors October 6, 1969 and, in revised form, February 2, 1970.

AMS 1969 subject classifications. Primary 1740.

Key words and phrases. Jordan algebra, quadratic Jordan algebra, representation, specialization, bimodule, extension, cohomology group, universal envelope.

Copyright (C) 1971, American Mathematical Society 
Basic consequences of the axioms are

(2) $V_{x, x}=V_{x^{2}}$

(3) $V_{x, 1}=V_{1, x}=U_{x, 1}=V_{x}$,

(4) $V_{x, y}=V_{x} V_{y}-U_{x, y}$,

(5) $2 U_{x}=V_{x}^{2}-V_{x^{2}}$.

A homomorphism $\varphi: \mathfrak{\Im} \rightarrow \mathfrak{\Im}$ of unital algebras is a linear map satisfying $\varphi(1)=\tilde{1}$, $\varphi\left(U_{x} y\right)=\tilde{U}_{\varphi(x)} \varphi(y)$. In the resulting category of unital quadratic Jordan algebras a subalgebra is a subspace $\Re$ with $1 \in \Re, U_{\Re} \Re \subset \Re$ and an ideal is a subspace with $U_{\Im} \Re \subset \Re, U_{\Re} \subset \Re$ (it follows from (4) and (3) that any Jordan product lies in $\Re$ if one of its factors does). A derivation in $\mathfrak{\Im}$ is a linear map $D: \Im \rightarrow \Im$ satisfying $D(1)=0$ and $D\left(U_{x} y\right)=U_{D x, x} y+U_{x} D y$.

One basic example of a unital quadratic Jordan algebra is the algebra $\mathfrak{A}^{+}$obtained from a unital associative algebra $\mathfrak{A}$ by introducing the Jordan structure

$$
1=1, \quad U_{x} y=x y x .
$$

Any (Jordan) subalgebra of such an algebra $\mathfrak{A}^{+}$is called a special Jordan algebra. A unital linear specialization of $\mathfrak{\Im}$ is a homomorphism $\sigma: \mathfrak{\Im} \rightarrow \mathfrak{A}^{+}$, i.e. a linear map satisfying

$$
\sigma(1)=1, \quad \sigma\left(U_{x} y\right)=\sigma(x) \sigma(y) \sigma(x) .
$$

If $\frac{1}{2} \in \Phi$ the category of unital quadratic Jordan algebras $(\mathfrak{X}, U, 1)$ is isomorphic to the category of unital linear Jordan algebras $(\mathfrak{X}, L, 1)$ (unital commutative linear algebras where the multiplication $x \cdot y=L_{x} y$ strictly satisfies the Jordan identity $\left[x^{2}, y, x\right]=0$, or $\left.\left[L_{x^{2}}, L_{x}\right]=0\right)$ by means of the formulas

$$
1=1, \quad U_{x}=2 L_{x}^{2}-L_{x^{2}}, \quad L_{x}=\frac{1}{2} V_{x}
$$

(compare with (5)). A unital representation of a unital linear Jordan algebra $\Im$ is a linear map $x \rightarrow \rho(x)$ of $\Im$ into some unital associative algebra such that

(UR I) $\rho(1)=1$,

(UR II) $\rho(x) \rho(y) \rho(x)+\rho\left(x^{2} \cdot y\right)=\rho\left(x^{2}\right) \rho(y)+\rho(x \cdot y) \rho(x)$,

(UR III) $\rho(x) \rho\left(x^{2}\right)=\rho\left(x^{2}\right) \rho(x)$

hold strictly. (For linear Jordan algebras see [3], for the quadratic case [4], [5].)

1. Multiplication algebras. If $\Im$ is a unital quadratic Jordan algebra over $\Phi$ and $X$ a unital subset of $\Im$ (in the sense that $1 \in X$ ) then the multiplication algebra $\mathscr{M}_{\Im}(X)$ determined by $X$ is the algebra of linear transformations on $\Im$ generated by the $U_{x}$ and $U_{x, y}$ for $x, y$ in $X$. (Where no misunderstanding can arise we abbreviate this as $\mathscr{M}(X)$.) Since $1 \in X$ and $U_{1}=I, \mathscr{M}(X)$ is a unital associative algebra. It is spanned by all operators of the form $M=M_{1} \ldots M_{n}$ where each $M_{i}$ is a $U_{x}$ or a $U_{x, y}$. If $X$ is a linear subset, so $x+y$ lies in $X$ whenever $x$ and $y$ do, then $U_{x, y}=$ $U_{x+y}-U_{x}-U_{y}$ shows that $\mathscr{M}(X)$ is generated by the $U_{x}$ alone. We also note that for $x, y$ in $X$ the operators $V_{x}$ and $V_{x, y}$ are contained in $\mathscr{M}(X)$ by (3) and (4). Thus $\mathscr{M}(X)$ contains all the $U$ 's and $V$ 's, that is, all the operators we could reasonably call "multiplications." 
If $\mathfrak{A}$ is any subspace of $\mathfrak{\Im}$ invariant under $\mathscr{M}(X)$ we denote by

$$
\mathscr{M}_{\Im}(X \mid \Re)=\left.\mathscr{M}_{\Im}(X)\right|_{\Re}
$$

the set of restrictions to $\AA$ of elements of $\mathscr{M}_{\Im}(X)$; this is a unital algebra of operators acting in $\Re$, and it is a homomorphic image of $\mathscr{M}(X)$.

The following closure property is basic:

THEOREM 1. If $X$ is a unital subset of a unital quadratic Jordan algebra then

$$
\mathscr{M}(\mathscr{M}(X) X)=\mathscr{M}(X) \text {. }
$$

Proof. Since $\mathscr{M}(X)=\mathscr{M}(Y)$ and $\mathscr{M}(X) X=\mathscr{M}(Y) Y$ for $Y$ the linear space spanned by $X$, it suffices to assume $X$ itself is linear. Thus $\mathscr{M}(X)$ is spanned by the $U_{x}$ 's.

Clearly $\mathscr{M}(X) \subset \mathscr{M}(\mathscr{M}(X) X)$ since $I \in \mathscr{M}(X)$ implies $X \subset \mathscr{M}(X) X$. To prove the converse we must show $U_{w}$ and $U_{z, w}$ are in $\mathscr{M}(X)$ if $z$ and $w$ are in $\mathscr{M}(X) X$. For this we recall the operator identity

$$
U_{U(x) y, z}=V_{x, y} U_{x, z}-U_{x} V_{y, z}=U_{x, z} V_{y, x}-V_{z, y} U_{x}
$$

(see [5, Lemma 1, (2.14)]). Let $Z=\left\{z \in \mathscr{M}(X) X \mid U_{z, x} \in \mathscr{M}(X)\right.$ for all $\left.x \in X\right\}$ and $Y=\left\{y \in \mathscr{M}(X) X \mid U_{y, z} \in \mathscr{M}(X)\right.$ for all $\left.z \in Z\right\}$. Clearly $X \subset Z$ by definition of $\mathscr{M}(X)$, so $Y \subset Z$ by definition of $Y$ and $Z$. By definition of $Z$ we also have $X \subset Y$, so

$$
X \subset Y \subset Z \subset \mathscr{M}(X) X
$$

where all these are linear sets. But $\mathscr{M}(X) Y \subset Y$ since if $x \in X, y \in Y$ we have $U_{x} y \in Y$ by (7). Thus $Y=Z=\mathscr{M}(X) X$. This shows that if $z, w$ are in $\mathscr{M}(X) X$ then $U_{z, w} \in U_{Y, Z} \subset \mathscr{M}(X)$. It also shows that $W=\left\{w \in \mathscr{M}(X) X \mid U_{w} \in \mathscr{M}(X)\right\}$ is a linear set containing $X$. But $\mathscr{M}(X) W \subset W$ since $U_{x} W \subset W$ by UQJ II. Thus $W=\mathscr{M}(X) X$ too, and all $U_{w}, U_{w, z}$ for $z, w$ in $\mathscr{M}(X) X$ belong to $\mathscr{M}(X)$.

This result has two important corollaries. If $\mathfrak{I}$ is a Jordan algebra over $\Phi$ and $X$ a unital subset of $\mathfrak{\Im}$ then the subalgebra $\Phi[X]$ generated by $X$ is the smallest subalgebra containing $X$. We say a subalgebra $\AA$ is generated by $X$ (or $X$ is a set of generators) if $\mathfrak{\Omega}=\Phi[X]$. (Note that we always assume $1 \in X$ is one of the generators.)

Corollary 2. $\Phi[X]=\mathscr{M}(X) X$.

Proof. Certainly $\Phi[X] \supset \mathscr{M}(X) X \supset X$, so it will suffice if $\mathscr{M}(X) X$ is a subalgebra: $z, w \in \mathscr{M}(X) X$ imply $U_{z} w \in \mathscr{M}(X) X$. But by the theorem $U_{z} \in \mathscr{M}(X)$, so $U_{z} w \in \mathscr{M}(X) \mathscr{M}(X) X=\mathscr{M}(X) X$.

Corollary 3. If $X$ generates $\AA$ then the $U_{x}$ and $U_{x, y}$ for $x, y$ in $X$ generate $\mathscr{M}(\Re): \mathscr{M}(\Re)=\mathscr{M}(X)$.

Proof. $\mathscr{M}(\mathfrak{\Re})=\mathscr{M}(\Phi[X])=\mathscr{M}(\mathscr{M}(X) X)=\mathscr{M}(X)$.

2. Representations, specializations, and bimodules. A multiplication representation of a unital quadratic Jordan algebra $\mathfrak{\Im}$ is a mapping of the form $\left.x \rightarrow U_{x}\right|_{\Re}$ of 
$\mathfrak{J}$ into $\mathscr{M}_{\mathfrak{F}}(\mathfrak{\Im} \mid \mathfrak{\Re})$ where $\mathfrak{F}$ is an (enveloping) Jordan algebra containing $\Im$, and $\mathfrak{A}$ is a subspace of $\leftleftarrows$ invariant under multiplication by $\Im$. These can be characterized abstractly as follows. A unital quadratic specialization of $\mathfrak{\Im}$ in a unital associative algebra $\mathfrak{A}$ is a quadratic mapping $\mu: \mathfrak{\Im} \rightarrow \mathfrak{A}$ strictly satisfying

(UQS I) $\mu(1)=1$,

(UQS II) $\mu\left(U_{x} y\right)=\mu(x) \mu(y) \mu(x)$,

(UQS III) $\mu\left(U_{x} y, x\right)=\mu(x) \nu(y, x)=\nu(x, y) \mu(x)$,

where, as in (1)-(5), $\mu(x, y)=\mu(x+y)-\mu(x)-\mu(y), \nu(x)=\mu(x, 1), \nu(x, y)=\nu(x) \nu(y)$ $-\mu(x, y)$. The first two conditions are just those of an ordinary linear specialization of $\Im$ in $\mathfrak{A}$, but since $\mu$ is quadratic rather than linear we require a third condition. A unital quadratic representation of $\mathfrak{\Im}$ on a $\Phi$-module $\mathfrak{M}$ is a unital quadratic specialization of $\mathfrak{\Im}$ in the algebra $\mathfrak{A}=\operatorname{Hom}_{\Phi}(\mathfrak{M}, \mathfrak{M})$. A unital bimodule for $\mathfrak{F}$ consists of a $\Phi$-module $\mathfrak{M}$ together with a unital quadratic representation of $\mathfrak{F}$ on $\mathfrak{M}$. Since any unital associative algebra may be imbedded in an algebra $\operatorname{Hom}_{\Phi}(\mathfrak{M}, \mathfrak{M})$ we see that quadratic specializations, quadratic representations, and bimodules are essentially equivalent concepts. Note also that the axioms UQS I-III on $\mu(x)$ are analogous to the axioms UQJ I-III on $U_{x}$. From this we see that any multiplication representation $\left.x \rightarrow U_{x}\right|_{\Re}$ of $\Im$ in $\mathscr{M}_{\mathfrak{S}}(\Im \mid \Re)$ furnishes a quadratic representation $x \rightarrow \mu(x)=\left.U_{x}\right|_{\Re}$ of $\mathfrak{\Im}$ on $\mathfrak{\Re}$. We will shortly prove that, conversely, every quadratic representation is obtained in this manner from a multiplication representation.

An important example is the regular representation $x \rightarrow U_{x}$ of $\mathfrak{I}$ on itself, and the corresponding regular bimodule.

By [5, Theorem 2] the condition UQS II on the product $U_{x} y$ can be replaced by the analogous conditions on the products $x^{2}$ and $x^{3}$.

THEOREM 4. A quadratic map $\mu: \mathfrak{I} \rightarrow \mathfrak{A}$ is a unital quadratic specialization if and only if

(UCS I) $\mu(1)=1$,

(UCS II) $\mu\left(U_{x} y, x\right)=\mu(x) \nu(y, x)=\nu(x, y) \mu(x)$,

(UCS III) $\mu\left(x^{2}\right)=\mu(x)^{2}$,

(UCS IV) $\mu\left(x^{3}\right)=\mu(x)^{3}$

hold strictly.

We now want to show that this notion of bimodule for the variety of unital quadratic Jordan algebras is equivalent to that due to Eilenberg [2]. Given a unital $\mathfrak{\Im}$-bimodule $\mathfrak{M}$ we can form the split null extension $\mathfrak{E}=\mathfrak{I} \oplus \mathfrak{M}$ with unit $1 \oplus 0$ and $U$-operator

$$
U_{x \oplus m}(y \oplus n)=U_{x} y \oplus\left\{\mu_{x}(n)+\nu_{x, y}(m)\right\}
$$

where $\mu_{x}=\mu(x), \nu_{x, y}=\nu(x, y)$ are determined by the unital quadratic representation of $\mathfrak{I}$ on $\mathfrak{M}$. The fundamental property of the split null extension is the 
Theorem 5 (Unital Bimodule TheORem). The split null extension \& of a unital quadratic Jordan algebra $\mathfrak{\Im}$ by a unital bimodule $\mathfrak{M}$ is again a unital quadratic Jordan algebra.

Proof. We must verify the Jordan axioms

(UQJ I) $U_{1 \oplus 0}(z \oplus p)=z \oplus p$,

(UQJ II) $U_{U(x \oplus m)(y \oplus n)}(z \oplus p)=U_{x \oplus m} U_{y \oplus n} U_{x \oplus m}(z \oplus p)$,

(UQJ III) $U_{U(x \oplus m)(y \oplus n), x \oplus m}(z \oplus p)=U_{x \oplus m} V_{y \oplus n, x \oplus m}(z \oplus p)=V_{x \oplus m, y \oplus n} U_{x \oplus m}(z \oplus p)$.

Now the components of these relations in $\Im$ are just the axioms UQJ I-III. For the components in $\mathfrak{M}$ we use the fact that $\mathfrak{M}$ is an ideal in $\mathfrak{E}$ and any product with two or more factors from $\mathfrak{M}$ is zero. The relations involving only a single element from $\mathfrak{M}$ are

(i) $\mu_{1}(p)=p$,

(ii) $\mu_{U(x) y}(p)=\mu_{x} \mu_{y} \mu_{x}(p)$,

(ii') $\nu_{U(x) y, z} \nu_{x, y}(m)=\mu_{x} \mu_{y} \nu_{x, z}(m)+\nu_{x, U(y) U(x) z}(m)$,

(ii") $\nu_{U(x) y, z} \mu_{x}(n)=\mu_{x} \nu_{y, U(x) z}(n)$,

(iii) $\mu_{U(x) y, x}(p)=\mu_{x} \nu_{y, x}(p)=\nu_{x, y} \mu_{x}(p)$,

(iii') $\nu_{U(x) y, z}(m)=\nu_{x, z} \nu_{x, y}(m)=\mu_{x} \mu_{y, z}(m)+\nu_{x,\{y x z\}}(m)=\nu_{x, y} \nu_{x, z}(m)+\nu_{U(x) z, y}(m)$,

(iii") $\nu_{x, z} \mu_{x}(n)=\mu_{x} \nu_{z, x}(n)=\mu_{U(x) z, x}(n)$.

Some of these are immediate: (i) is just UQS I, (ii) is UQS II, (iii) and (iii") are UQS III. To establish (ii"), linearize UQS III (which is possible since it holds strictly) to obtain $\mu\left(U_{x} y, U_{x} z\right)+\mu\left(U_{x, U(x) z} y, x\right)=\mu(x) \nu\left(y, U_{x} z\right)+\mu\left(U_{x} z, x\right) \nu(y, x)=$ $\nu(x, y) \mu\left(U_{x} z, x\right)+\nu\left(U_{x} z, y\right) \mu(x)$. Since the first sum is symmetric in $y$ and $z$ ( $\left\{U_{x} z \quad y \quad x\right\}=U_{x}\left\{\begin{array}{lll}y & x & z\end{array}\right\}$ by UQJ III) the second and third are too, so $\mu(x) \nu\left(y, U_{x} z\right)$ $+\nu(x, z) \mu(x) \nu(y, x)=\nu(x, z) \mu(x) \nu(y, x)+\nu\left(U_{x} y, z\right) \mu(x)$ (by UQS III), and (ii") holds.

For (ii') and (iii') we need the identities

$$
\begin{gathered}
\nu(x, y) \nu(x, z)=\nu\left(U_{x} y, z\right)+\mu(x) \mu(y, z), \\
\nu(x, y) \nu(z, y)=\nu\left(x, U_{y} z\right)+\mu(x, z) \mu(y) \\
\nu\left(U_{x} y, y\right)=\nu\left(x, U_{y} x\right) .
\end{gathered}
$$

These will follow from the analogue of (7),

$$
\mu\left(U_{x} y, z\right)=\nu(x, y) \mu(x, z)-\mu(x) \nu(y, z)=\mu(x, z) \nu(y, x)-\nu(z, y) \mu(x)
$$

which holds by [5, Lemma 1, (2.14)]. Thus from (10) we see

$$
\begin{aligned}
\nu\left(U_{x} y, z\right)+\mu(x) \mu(y, z) & =\nu\left(U_{x} y\right) \nu(z)-\mu\left(U_{x} y, z\right)+\mu(x) \mu(y, z) \\
& =\mu\left(U_{x} y, 1\right) \nu(z)-\{\nu(x, y) \mu(x, z)-\mu(x) \nu(y, z)\}+\mu(x) \mu(y, z) \\
& =\{\nu(x, y) \mu(x, 1)-\mu(x) \nu(y, 1)\} \nu(z)-\nu(x, y) \mu(x, z) \\
& +\nu(x)\{\nu(y, z)+\mu(y, z)\} \\
& =\nu(x, y)\{\nu(x) \nu(z)-\mu(x, z)\}-\mu(x) \nu(y) \nu(z)+\mu(x)\{\nu(y) \nu(z)\} \\
& =\nu(x, y) \nu(x, z) .
\end{aligned}
$$


The second part of (8) follows similarly. Then $\nu\left(U_{x} y, y\right)=\nu(x, y) \nu(x, y)-\mu(x) \mu(y, y)$ $=\nu(x, y) \nu(x, y)-2 \mu(x) \mu(y)=\nu(x, y) \nu(x, y)-\mu(x, x) \mu(y)=\nu\left(x, U_{y} x\right)$ by setting $z=y$ in the first part of (8) and $z=x$ in the second. This establishes (9).

Once we have (8) and (9) we finish (ii'), (iii') as follows. The first part of (iii') comes from $\mu(x) \mu(y, z)+\nu(x,\{y x z\})-\nu\left(U_{x} y, z\right)-\nu(x, z) \nu(x, y)=\nu\left(x, U_{y, z} x\right)-$ $\nu\left(U_{x} y, z\right)-\nu\left(U_{x} z, y\right)=0$ by (8) and linearized (9). The second part of (iii') follows by symmetry. For (ii'), $\mu(x) \mu(y) \nu(x, z)+\nu\left(x, U_{y} U_{x} z\right)-\nu\left(U_{x} y, z\right) \nu(x, y)=\mu(x) \mu(y) \nu(x, z)$ $+\left\{\nu(x, y) \nu\left(U_{x} z, y\right)-\mu\left(x, U_{x} z\right) \mu(y)\right\}-\{\nu(x, y) \nu(x, z)-\mu(x) \mu(y, z)\} \nu(x, y)$ (by (8)) $=\mu(x)\{\mu(y) \nu(x, z)-\nu(z, x) \mu(y)+\mu(y, z) \nu(x, y)\}+\nu(x, y)\left\{\nu\left(U_{x} z, y\right)-\nu(x, z) \nu(x, y)\right\}$ (by UQS III) $=\mu(x)\{\nu(y, x) \mu(y, z)\}+\nu(x, y)\{-\mu(x) \mu(y, z)\}$ (by (8) and linearized UQS III) $=0$ by UQS III.

We have already noted the equivalence of the concepts of quadratic specialization, quadratic representation, and bimodule. The Bimodule Theorem now shows that these are equivalent in turn to the notion of a multiplication representation, since the quadratic representation $x \rightarrow \mu(x)$ of $\mathfrak{I}$ in $\operatorname{Hom}_{\Phi}(\mathfrak{M}, \mathfrak{M})$ coincides with the multiplication representation $\left.x \rightarrow U_{x}\right|_{\mathfrak{M}}$ of $\mathfrak{S}$ in $\mathscr{M}_{\mathfrak{E}}(\mathfrak{\Im} \mid \mathfrak{M})$ (in $\mathfrak{E}$ we have $U_{x} m=\mu_{x} m$ for $x \in \mathfrak{J}, m \in \mathfrak{M}$, i.e. $\left.\mu(x)=U_{x} \mid \mathfrak{m}\right)$.

We say two unital quadratic specializations $\mu: \mathfrak{\Im} \rightarrow \mathfrak{A}, \tilde{\mu}: \mathfrak{\Im} \rightarrow \tilde{\mathfrak{A}}$ are equivalent if there is an isomorphism $\varphi: \mathfrak{A} \rightarrow \tilde{\mathfrak{A}}$ with $\tilde{\mu}=\varphi \circ \mu$. Similarly two representations $\mu, \tilde{\mu}$ of $\mathfrak{\Im}$ on $\mathfrak{M}, \tilde{\mathfrak{M}}$ are equivalent if there is a linear bijection $\psi: \mathfrak{M} \rightarrow \tilde{\mathfrak{M}}$ with $\tilde{\mu}(x)=\psi \circ \mu(x) \circ \psi^{-1}$ (if $\Phi$ is a field any isomorphism $\varphi: \mathfrak{A}=\operatorname{Hom}_{\Phi}(\mathfrak{M}, \mathfrak{M}) \rightarrow \tilde{\mathfrak{A}}$ $=\operatorname{Hom}_{\Phi}(\tilde{\mathfrak{M}}, \tilde{\mathfrak{M}})$ has the form $\varphi(a)=\psi \circ a \circ \psi^{-1}$ for such a $\left.\psi\right)$. In this case we say $\psi: \mathfrak{M} \rightarrow \tilde{\mathfrak{M}}$ is an isomorphism of unital $\mathfrak{\Im}$-bimodules. More generally, $\psi$ is a homomorphism of $\mathfrak{\Im}$-bimodules if

$$
\psi(\mu(x) m)=\tilde{\mu}(x) \psi(m) \quad(x \in \mathfrak{J}, m \in \mathfrak{M}) .
$$

Our results also show that if $\frac{1}{2} \in \Phi$ the concepts of a quadratic representation $\mu$ for a quadratic Jordan algebra and a linear representation $\rho$ for a linear Jordan algebra are equivalent by means of

$$
\mu(x)=2 \rho(x)^{2}-\rho\left(x^{2}\right), \quad \rho(x)=\frac{1}{2} \nu(x)
$$

(compare (6)). Indeed, if $\mu$ is a quadratic representation of $\mathfrak{\Im}$ on $\mathfrak{M}$ then $\mathfrak{E}=\mathfrak{\Im} \oplus \mathfrak{M}$ is a quadratic Jordan algebra, so if $\frac{1}{2} \in \Phi$ by (6) it is also a linear Jordan algebra relative to $L_{x}=\frac{1}{2} V_{x}$, and we have a linear representation of $\mathfrak{J}$ on $\mathfrak{M}$ by $\rho(x)=\left.L_{x}\right|_{\mathfrak{M}}$ $=\left.\frac{1}{2} V_{x}\right|_{\mathfrak{M}}=\frac{1}{2} \nu(x)$. Conversely, if $\rho$ is a linear representation $\mathfrak{E}=\mathfrak{F} \oplus \mathfrak{M}$ is a linear Jordan algebra, hence a quadratic Jordan algebra under $U_{x}=2 L_{x}^{2}-L_{x^{2}}$, and the corresponding quadratic representation on the bimodule $\mathfrak{M}$ is

$$
\mu(x)=\left.U_{x}\right|_{\mathfrak{M}}=\left.\left(2 L_{x}^{2}-L_{x^{2}}\right)\right|_{\mathfrak{M}}=2 \rho(x)^{2}-\rho\left(x^{2}\right) .
$$

Since quadratic specializations are not linear maps we cannot define the kernel simply to be the set of elements mapped into zero. One of the basic properties of the kernel $\mathfrak{R}$ in the linear case is that the map is constant on the cosets of $\Re$. If this 
is to be true also in the quadratic case, $\mu(x+\Re)=\mu(x)$, we must have $\mu(\Re)=0$ (setting $x=0$ ) and $\mu(x, \Re)=\mu(x+\Re)-\mu(x)-\mu(\Re)=0$. Thus we are led to define the kernel of a quadratic specialization $\mu$ to be

$$
\operatorname{Ker} \mu=\{z \mid \mu(z)=\mu(z, x)=0 \text { for all } x \in \Im\} .
$$

(This is just the usual radical of the quadratic map $\mu$.) For such $z$ we have $\nu(z)$ $=\nu(z, x)=\nu(x, z)=0$. The kernel $\mathfrak{R}$ is a subspace since the condition $\mu(z, \Im)=0$ is linear in $z$ and it guarantees $\mu(z)=0$ will be linear since $\mu\left(z+z^{\prime}\right)=\mu(z)+\mu\left(z, z^{\prime}\right)$

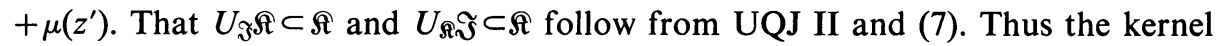
is always an ideal. The kernel of the regular representation is called the extreme radical, or simply the kernel, of the algebra $\Im$. (Since $U_{z, 1} 1=z \circ 1=2 z$ is zero for $z$ in the extreme radical 3 we have $28=0$, so if $\frac{1}{2} \in \Phi$ there is no extreme radical.)

We say a quadratic specialization is faithful if its kernel is zero (this does not imply it is injective as a map). Given $\mu: \mathfrak{J} \rightarrow \mathfrak{A}$ we can always obtain a faithful representation $\bar{\mu}: \overline{\mathfrak{J}} \rightarrow \mathfrak{A}$ for $\overline{\mathfrak{\Im}}=\mathfrak{\Im} / \operatorname{Ker} \mu$ by $\bar{\mu}(\bar{x})=\mu(x)$. Note that a representation of a simple algebra is either faithful or zero.

In the case of a linear representation $\rho$ of a linear Jordan algebra the kernel is defined in the usual way as the set of $z$ for which $\rho(z)=0$. Unfortunately, this kernel need not be an ideal, only an associator ideal. We are suggesting that the proper definition of the kernel of $\rho$ should be the set of $z$ for which $\rho(z)$ and all $\rho(z \cdot x)$ are zero. Indeed,

Proposition 6. If $\frac{1}{2} \in \Phi$ then the kernel of a unital quadratic representation $\mu$ is $\{z \mid \rho(z)=\rho(z \cdot x)=0$ for all $x\}$.

Proof. Such $z$ certainly belong to the kernel of $\mu$ since by (6) $\mu(z)=2 \rho(z)^{2}-\rho(z \cdot z)$ and $\mu(x, z)=2\{\rho(z) \rho(x)+\rho(x) \rho(z)-\rho(z \cdot x)\}$. Conversely, if $z$ belongs to the kernel of $\mu$ then $\rho(z)=\frac{1}{2} \nu(z)=\frac{1}{2} \mu(z, 1)=0$ and $\rho(z \cdot x)=\rho(z) \rho(x)+\rho(x) \rho(z)-\frac{1}{2} \mu(z, x)=0$.

3. Cohomology. Let $\mathfrak{\Im}$ be a unital quadratic Jordan algebra and $\mathfrak{M}$ a unital $\Im$-bimodule. A derivation of $\mathfrak{I}$ in $\mathfrak{M}$ is a linear map $d$ satisfying

$$
d(1)=0, \quad d\left(U_{x} y\right)=\mu(x) d(y)+\nu(x, y) d(x) .
$$

For example, if $x \in \mathfrak{\Im}, m \in \mathfrak{M}$ then

$$
d_{x, m}(y)=\mu(x, y) m-\nu(y, x) m
$$

defines such a derivation of $\mathfrak{I}$ in $\mathfrak{M}$. In terms of the operations in the split null extension $\mathfrak{E}=\mathfrak{I} \oplus \mathfrak{M}$ this becomes

$$
d_{x, m}(y)=\left\{\begin{array}{lll}
x & m & y
\end{array}-\{m x y\}=\left\{V_{x, m}-V_{m, x}\right\} y=\left[V_{x}, V_{m}\right] y .\right.
$$

Any linear combination of such $d_{x, y}$ 's will be called an inner derivation. The derivations of $\mathfrak{J}$ in $\mathfrak{M}$ form a linear space $\operatorname{Der}(\mathfrak{S}, \mathfrak{M})$ with the inner derivations Inder $(\mathfrak{I}, \mathfrak{M})$ as a subspace. The quotient

$$
H^{1}(\Im, \mathfrak{M})=\operatorname{Der}(\Im, \mathfrak{M}) / \operatorname{Inder}(\Im, \mathfrak{M})
$$


is the 1st cohomology group. Thus the vanishing of the first cohomology group is equivalent to the condition that all derivations be inner.

We can express $H^{1}$ in terms of cocycles and coboundaries. The space $C^{1}(\mathfrak{I}, \mathfrak{M})$ of all 1-cochains consists of all linear maps $f: \mathfrak{I} \rightarrow \mathfrak{M}$ such that $f(1)=0$. Such an $f$ belongs to the space $Z^{1}(\mathfrak{\Im}, \mathfrak{M})$ of 1-cocycles if

$$
\left\{\partial^{1} f\right\}(x ; y)=\mu(x) f(y)+\nu(x, y) f(x)-f\left(U_{x} y\right)
$$

is zero for each $x$ and $y$ in $\Im$, and it belongs to the 1-coboundaries $B^{1}(\Im, \mathfrak{M})$ if it is of the form $f=\partial^{0} g$ for some $g=\sum x_{i} \otimes m_{i}$ in $\mathfrak{M} \otimes \mathfrak{M}$, where

$$
\left\{\partial^{\circ}(x \otimes m)\right\}(y)=\mu(x, y) m-\nu(y, x) m .
$$

The maps $\partial^{0}$ and $\partial^{1}$ have been chosen so that we have $Z^{1}(\mathfrak{I}, \mathfrak{M})=\operatorname{Der}(\mathfrak{I}, \mathfrak{M})$, $B^{1}(\Im, \mathfrak{M})=\operatorname{Inder}(\mathfrak{S}, \mathfrak{M})$, and hence the usual

$$
H^{1}(\Im, \mathfrak{M})=Z^{1}(\Im, \mathfrak{M}) / B^{1}(\Im, \mathfrak{M}) .
$$

If we take $C^{0}(\mathfrak{I}, \mathfrak{M})=\mathfrak{I} \otimes \mathfrak{M}$ then $Z^{0}(\mathfrak{I}, \mathfrak{M})$ should be the kernel of the above $\partial^{0}$, i.e. all $\sum x_{i} \otimes m_{i}$ satisfying the commutativity conditions

$$
\sum\left\{x_{i} m_{i} y\right\}=\sum\left\{m_{i} x_{i} y\right\} \text { or }\left[V_{x_{i}}, V_{m_{i}}\right] y=0
$$

for all $y$. All $1 \otimes m$ trivially belong to $Z^{0}$ since $V_{1}=2 I$. It is natural to disregard the space $B^{\circ}(\mathfrak{I}, \mathfrak{M})=1 \otimes \mathfrak{M}$ of such 0 -cycles, and hence regard

$$
H^{0}(\Im, \mathfrak{M})=Z^{0}(\Im, \mathfrak{M}) / B^{0}(\Im, \mathfrak{M})
$$

as a measure of the commutativity of the representation of $\mathfrak{\Im}$ on $\mathfrak{M}$. Thus $H^{0}$ being zero is equivalent to

$$
\{x y m\}=\{x m y\}
$$

for all $x, y, m$.

To define a second cohomology group $H^{2}(\Im, \mathfrak{M})$ we introduce the concept of an extension of $\mathfrak{I}$ by $\mathfrak{M}$, which is an exact sequence

$$
0 \longrightarrow \mathfrak{M} \stackrel{i}{\longrightarrow} \mathfrak{x} \stackrel{\pi}{\longrightarrow} \mathfrak{\Im} 0
$$

where $\mathfrak{E}$ and $\mathfrak{S}$ are unital quadratic Jordan algebras, $\mathfrak{M}$ simply a quadratic Jordan algebra, and $i, \pi$ are homomorphisms. We henceforth identify $\mathfrak{M}$ with $i(\mathfrak{M})$, so regard $\mathfrak{M}$ as an ideal contained in $\mathbb{E}$. If $\mathfrak{M}$ is null (all products zero) we speak of a null extension. In this case $\mathfrak{M}$ has a natural $\Im$-bimodule structure: as an ideal $\mathfrak{M}$ is a unital $\mathfrak{E}$-bimodule via the regular representation, and the kernel of this representation contains $\mathfrak{M}$ since $\mathfrak{M}$ is null, so we have an induced representation of $\mathfrak{E} / \mathfrak{M} \cong \mathfrak{I}$ on $\mathfrak{M}$. The bimodule action is given (for any set-theoretic lifting $\alpha: \mathfrak{I} \rightarrow \mathfrak{F}$ ) by

$$
\mu(x) m=U_{\alpha(x)} m, \nu(x, y) m=\{\alpha(x) \alpha(y) m\} \quad(\pi \alpha(z)=z) .
$$


An extension is split if there is a lifting $\alpha: \mathfrak{\Im} \rightarrow \mathfrak{E}$ which is an algebra homomorphism satisfying $\pi \circ \alpha=1 \mathfrak{3}$; this means $\mathfrak{F}=\alpha(\Im) \oplus \mathfrak{M}$ for $\alpha(\Im)$ a subalgebra isomorphic to $\Im$ under $\pi$ (justifying our earlier use of "split null extension"). We have the usual notion of equivalence of extensions,

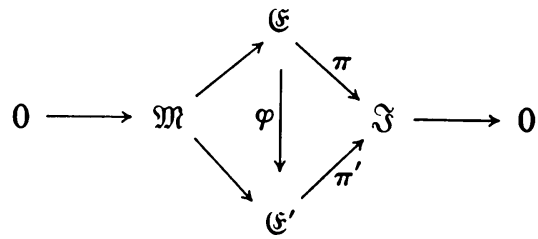

or that $\varphi$ is an algebra isomorphism which is the identity on $\mathfrak{M}$ and satisfies $\pi^{\prime} \circ \varphi=\pi$.

We now restrict our attention to those null extensions which are linearly split in the sense that there exist linear liftings $\alpha: \Im \rightarrow \notin$ with $\pi \circ \alpha=1_{\mathfrak{S}}$ and such that $\alpha(1)=1$ (if $\Phi$ is a field all extensions are linearly split). Since $\mathfrak{M}$ is a null ideal and the bimodule action is given by (13), we have $\mathfrak{E}=\alpha(\Im) \oplus \mathfrak{M}$ as a linear space with multiplication

$$
U_{\alpha(x) \oplus m} \alpha(y) \oplus n=\alpha\left(U_{x} y\right) \oplus\{q(x ; y)+\mu(x) n+\nu(x, y) m\}
$$

where

$$
q(x ; y)=U_{\alpha(x)} \alpha(y)-\alpha\left(U_{x} y\right) \in \mathfrak{M}
$$

since $\pi$ is a homomorphism and $\pi \circ \alpha=1 \mathfrak{r}$ so $\pi q(x ; y)=0$. The condition that this multiplication satisfy the Jordan axioms UQJ I-III reduces to the condition that $\mathfrak{M}$ be a unital $\Im$-bimodule relative to $\mu$ and that $U_{\alpha(1)} \alpha(y)=\alpha(y), U_{\alpha(U(x) y)} \alpha(z)$ $=U_{\alpha(x)} U_{\alpha(y)} U_{\alpha(x)} \alpha(z), U_{\alpha(U(x) y), \alpha(x)} \alpha(z)=U_{\alpha(x)}\{\alpha(y) \alpha(x) \alpha(z)\}$. These amount to

$$
q(1 ; y)=0,
$$

(ii) $q\left(U_{x} y ; z\right)+\left\{U_{x} y z q(x ; y)\right\}=q\left(x ; U_{y} U_{x} z\right)+U_{x} q\left(y ; U_{x} z\right)+U_{x} U_{y} q(x ; z)$,

(iii) $q\left(x, U_{x} z ; y\right)+\{x y q(x ; z)\}=q(x ;\{y x z\})+U_{x} q(y, z ; x)$,

where $q(x, y ; z)=q(x+y ; z)-q(x ; z)-q(y ; z)$, and that these remain valid under all scalar extensions (or their linearizations hold in the original algebra).

If $\alpha^{\prime}$ is another lifting of $\Im$ into $\&$ then $\alpha^{\prime}=\alpha+f$ where $f(\Im) \subset \operatorname{Ker} \pi=\mathfrak{M}$. The corresponding map is

$$
\begin{aligned}
q^{\prime}(x ; y) & =U_{\alpha(x)+f(x)}\{\alpha(y)+f(y)\}-\alpha\left(U_{x} y\right)-f\left(U_{x} y\right) \\
& =U_{\alpha(x)} \alpha(y)+U_{\alpha(x)} f(y)+\{\alpha(x) \alpha(y) f(x)\}-\alpha\left(U_{x} y\right)-f\left(U_{x} y\right) \\
& =q(x ; y)+\mu(x) f(y)+\nu(x, y) f(x)-f\left(U_{x} y\right) \quad(\text { by }(13)) \\
& =q(x ; y)+\partial^{1} f(x ; y)
\end{aligned}
$$

as in (11). 
Any $q: \mathfrak{\Im} \times \mathfrak{I} \rightarrow \mathfrak{M}$ such that $q(x ; y)$ is quadratic in $x$ and linear in $y$ and satisfies (15) strictly is called a factor set of $\mathfrak{\Im}$ in the bimodule $\mathfrak{M}$. We say two factor sets are equivalent if they differ by a coboundary $\partial^{1} f$ as above. The equivalence classes form a space $H^{2}(\Im, \mathfrak{M})$ called the 2nd cohomology group. We have shown that any null extension of $\mathfrak{\Im}$ by the bimodule $\mathfrak{M}$ (i.e. such that the bimodule action given by (13) coincides with the given bimodule structure) determines an element of $H^{2}(\mathfrak{J}, \mathfrak{M})$. All elements of $H^{2}$ arise in this fashion, since from a factor set (15) we construct an extension (14) which obviously has $U_{x \oplus 0}(y \oplus 0)-\left(U_{x} y\right) \oplus 0=q(x, y)$ the given factor set.

If $\mathfrak{F}^{\prime}$ is equivalent to $\Subset$ under $\varphi, \alpha$ a lift of $\Im$ into $\mathfrak{E}$, then $\alpha^{\prime}=\varphi \circ \alpha$ is a lift of $\Im$ into $\mathbb{E}^{\prime}$ and

$$
\begin{aligned}
q^{\prime}(x ; y) & =U_{\alpha^{\prime}(x)}^{\prime} \alpha^{\prime}(y)-\alpha^{\prime}\left(U_{x} y\right) \\
& =\varphi\left\{U_{\alpha(x)} \alpha(y)-\alpha\left(U_{x} y\right)\right\} \\
& =\varphi\{q(x ; y)\} \\
& =q(x ; y)
\end{aligned}
$$

since $\varphi$ is the identity on $\mathfrak{M}$. Thus equivalent extensions determine the same equivalence class of factor sets. Conversely, if $\mathfrak{F}=\alpha(\mathfrak{\Im}) \oplus \mathfrak{M}$ and $\mathfrak{F}^{\prime}=\alpha^{\prime}(\mathfrak{\Im}) \oplus \mathfrak{M}$ are extensions such that their factor sets $q, q^{\prime}$ are equivalent, $q^{\prime}=q+\partial^{1} f$, then we can replace the lift $\alpha$ by $\alpha+f($ note $\alpha(1)+f(1)=1$ since $f(1)=0)$ and thus assume the lifts $\alpha, \alpha^{\prime}$ are chosen so that $q^{\prime}=q$. Then by (14) it is clear that $\varphi(\alpha(x) \oplus m)=$ $\alpha^{\prime}(x) \oplus m$ define an isomorphism of $\mathfrak{E}^{-}$onto $\mathbb{E}^{\prime}$ with $\varphi=I$ on $\mathfrak{M}$ and $\pi^{\prime} \circ \varphi=\pi$, so (5) and $\&^{\prime}$ are equivalent.

Thus we have a 1-1 correspondence between equivalence classes of linearly split null extensions of $\mathfrak{\Im}$ by the bimodule $\mathfrak{M}$, and the space $H^{2}(\mathfrak{I}, \mathfrak{M})$ of equivalence classes of factor sets of $\mathfrak{\Im}$ in $\mathfrak{M}$. The condition that $H^{2}(\mathfrak{M}, \mathfrak{M})$ be zero is that all linearly split null extensions are split in the sense of algebras. (An extension is split if and only if its factor set is equivalent to the zero factor set.)

We could also formulate this in terms of cocycles and coboundaries. We let $C^{2}(\mathfrak{M}, \mathfrak{M})$ be all maps $q: \mathfrak{I} \times \mathfrak{\Im} \rightarrow \mathfrak{M}$ such that $q(x ; y)$ is linear in $y$ and quadratic in $x$ and $q(1 ; y) \equiv 0$. Naturally $B^{2}(\Im, \mathfrak{M})$ will consist of all coboundaries $\partial^{1} f$ for $f$ in $C^{1}(\mathfrak{I}, \mathfrak{M})$. The cocycles $Z^{2}(\Im, \mathfrak{M})$ will be all cochains $q$ for which

$$
\begin{gathered}
\left\{\partial_{1}^{2} q\right\}(x ; y ; z)=q\left(U_{x} y ; z\right)+\nu\left(U_{x} y, z\right) q(x ; y)-q\left(x ; U_{y} U_{x} z\right) \\
-\mu(x) q\left(y ; U_{x} z\right)-\mu(x) \mu(y) q(x ; z), \\
\left\{\partial_{2}^{2} q\right\}(x ; y ; z)=q\left(x, U_{x} z ; y\right)+\nu(x, y) q(x ; z)-q(x ;\{y x z\}) \\
-\mu(x) q(y, z ; x)
\end{gathered}
$$

are zero and remain zero in all scalar extensions (equivalently we could define linearizations of $\partial_{1}^{2}$ and $\left.\partial_{2}^{2}\right)$. Then

as desired.

$$
H^{2}(\mathfrak{M}, \mathfrak{M})=Z^{2}(\mathfrak{S}, \mathfrak{M}) / B^{2}(\Im, \mathfrak{M})
$$


For a fixed algebra $\Im$ we obtain a functor $H^{n}(n=0,1,2)$ from the category of unital $\mathfrak{\Im}$-bimodules to the category of $\Phi$-modules by associating to a bimodule $\mathfrak{M}$ the space $H^{n}(\Im, \mathfrak{M})$ and to a morphism $\psi: \mathfrak{M} \rightarrow \mathfrak{\mathfrak { M }}$ the morphism

$$
H^{n}(\psi): H^{n}(\mathfrak{\Im}, \mathfrak{M}) \rightarrow H^{n}(\mathfrak{\Im}, \mathfrak{\mathfrak { M }})
$$

obtained from the following cochain maps: take $\psi^{0}=1 \otimes \psi$ for $C^{0}=\mathfrak{I} \otimes \mathfrak{M}$ $\rightarrow \mathfrak{\Im} \otimes \tilde{\mathfrak{M}}=\tilde{C}^{0}$ and take $\psi^{i}: C^{i} \rightarrow \tilde{C}^{i}$ by $f \rightarrow \tilde{f}=\psi \circ f$ or $q \rightarrow \tilde{q}=\psi \circ q$ when $i=1,2$. These maps commute with coboundary maps by the formulas (11), (12), (16) and the fact that $\psi$ is a morphism of bimodules.

Another basic property is the linearity of $H^{n}$ : it is additive,

$$
H^{n}\left(\Im, \bigoplus_{i} \mathfrak{M}_{i}\right)=\bigoplus_{i} H^{n}\left(\Im, \mathfrak{M}_{i}\right),
$$

and homogeneous,

$$
H^{n}\left(\Im_{\Omega}, \mathfrak{M}_{\Omega}\right)=H^{n}(\mathfrak{I}, \mathfrak{M})_{\Omega}
$$

as long as $\Omega$ is a free extension of $\Phi$-e.g. if $\Phi \subset \Omega$ are fields.

4. Universal gadgets. We can define a universal object for unital quadratic specializations just as for linear ones [4, p. 1073]. A universal unital quadratic envelope for a unital quadratic Jordan algebra $\Im$ consists of a unital associative algebra $\mathscr{U} \mathscr{Q} \mathscr{E}(\Im)$ together with a universal unital quadratic specialization $u$ : $\Im$ $\rightarrow \mathscr{U} \mathscr{Q} \mathscr{E}(\mathfrak{\Im})$ such that any unital quadratic specialization factors through $u$,

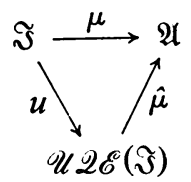

where $\hat{\mu}$ is a homomorphism of unital associative algebras. $\mathscr{U} \mathscr{Q} \mathscr{E}(\mathfrak{\Im})$ exists and is unique up to isomorphism (see $[1$, p. 182]).

The general philosophy behind universal gadgetry is that to prove a result about all something-or-others it suffices to prove it for the universal something-or-other. For example, the universal quadratic envelope reduces the investigation of all possible quadratic specializations of $\mathfrak{\Im}$ (or all possible bimodules) to the study of the single associative algebra $\mathscr{U} \mathscr{Q} \mathscr{E}(\Im)$. If $\mathfrak{\Im}$ is finite dimensional over a field we will see that $\mathscr{U} \mathscr{Q} \mathscr{E}(\Im)$ is also finite dimensional, and thus amenable to analysis.

We turn the universal quadratic specialization $u ; \Im \rightarrow \mathscr{U} \mathscr{Q} \mathscr{E}(\Im)$ into a universal unital quadratic representation $x \rightarrow u(x) \rightarrow L_{u(x)}$ of $\mathfrak{\Im}$ in $\operatorname{Hom}_{\Phi}(\mathscr{U} \mathscr{Q} \mathscr{E}(\Im), \mathscr{U} \mathscr{Q} \mathscr{E}(\Im))$ by means of the left regular representation $a \rightarrow L_{a}$ of $\mathscr{U} \mathscr{2} \mathscr{E}(\Im)$. This in turn converts $\mathscr{U} \mathscr{Q} \mathscr{E}(\Im)$ into a $\Im$-bimodule, the universal unital bimodule $\mathfrak{M}_{0}$ for $\mathfrak{~}$. It is not quite true that every unital bimodule $\mathfrak{M}$ is a homomorphic image of $\mathfrak{M}_{0}: \mathfrak{M}$ may be too large. However, every $\mathfrak{M}$ is a homomorphic image of some direct sum of copies of the universal bimodule. This is true because any cyclic unital $\Im$-bimodule 
is a homomorphic image of $\mathfrak{M}_{0}$ (so we perhaps should call it the universal cyclic

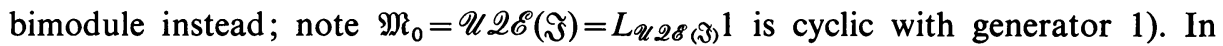
universal diagrams this means that any representation $\mu$ of $\Im$ on a cyclic bimodule $\mathfrak{M}$ with generator $m$ factors uniquely through the universal representation $u_{0}$ by means of a morphism $\psi$ of bimodules sending the generator 1 into the generator $m$ :

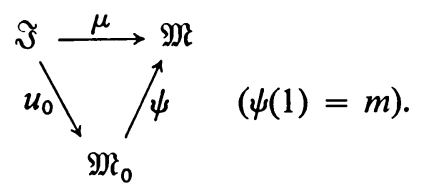

To see this, note $\mu: \mathfrak{\Im} \rightarrow \operatorname{Hom}_{\Phi}(\mathfrak{M}, \mathfrak{M})$ extends to an associative homomorphism $\hat{\mu}: \mathscr{U} \mathscr{Q} \mathscr{E}(\mathfrak{I}) \rightarrow \operatorname{Hom}_{\Phi}(\mathfrak{M}, \mathfrak{M})$. We get a linear map $\psi: \mathfrak{M}_{0}=\mathscr{U} \mathscr{Q} \mathscr{E}(\mathfrak{I}) \rightarrow \mathfrak{M}$ by $\psi(a)=\hat{\mu}(a) m$. Then $\psi(1)=m, \psi$ is surjective since $\mathfrak{M}$ is cyclic, and it is a morphism of bimodules since $\psi\left(u_{x}(a)\right)=\psi\left(L_{u(x)} a\right)=\psi(u(x) a)=\hat{\mu}(u(x) a) m=\hat{\mu}(u(x)) \hat{\mu}(a) m=$ $\mu(x) \hat{\mu}(a) m=\mu(x) \psi(a)$ using $\hat{\mu} \circ u=\mu$.

Note that because of this, to prove some identity for all unital quadratic specializations (i.e. for $\mathscr{U} \mathscr{L} \mathscr{E}(\Im)$ ) it suffices to establish it in all unital §-bimodules $\mathfrak{M}$, or in the Jordan algebra $\mathbb{E}=\mathfrak{I} \oplus \mathfrak{M}$. Thus relations holding in all algebras hold for all specializations.

As another application of universal gadgetry, by the homomorphism and additivity properties (17) and (18) of the cohomology functors, if the cohomology $H^{n}\left(\mathfrak{J}, \mathfrak{M}_{0}\right)$ vanishes for the universal $\mathfrak{\Im}$-bimodule $\mathfrak{M}_{0}$ it will vanish for any bimodule $\mathfrak{M}$.

One establishes the functorial properties of $\mathscr{U} \mathscr{L} \mathscr{E}(\Im)$ exactly as for the universal linear envelope $\mathscr{U} \mathscr{L} \mathscr{E}(\mathfrak{J})$ (see [3, pp. 96-99]). In particular, $\mathscr{U} \mathscr{\mathscr { E }}$ commutes with scalar extensions and direct limits.

Unlike the case of linear Jordan algebras, the universal unital quadratic specialization $u: \mathfrak{\Im} \rightarrow \mathscr{U} \mathscr{\mathscr { E }} \mathscr{E}(\Im)$ need not be faithful. If $\mathfrak{\Im}$ is the "standard counterexample" $\left\{1, z, z^{3}\right\}$ with $z^{2}=0$ but $z^{3} \neq 0$ (i.e. $\Im=\Phi[x] / \Omega$ for $\Re$ the Jordan ideal spanned by $\left.x^{2}, 2 x^{3}, x^{4}, x^{5} \ldots\right)$ then $z^{2}=0$ implies $\mu\left(z^{3}\right)=\mu\left(z^{2}\right) \mu(z)=0, \mu\left(z^{3}, 1\right)$ $=\nu\left(z^{3}\right)=\nu\left(z, z^{2}\right)=0, \mu\left(z^{3}, z\right)=\mu(z) \nu\left(z^{2}\right)=0$ so $\mu\left(z^{3}\right)=\mu\left(z^{3}, x\right)=0$ for all $x$ and $z^{3}$ is a nonzero element in the kernel of any unital quadratic specialization (in particular for $u$ ). Of course, $u$ will be faithful if the regular representation $U$ is faithful, i.e. $\Im$ has no extreme radical; this will always be the case if $\Im$ is semisimple or if $\frac{1}{2} \in \Phi$.

It is well known that the universal linear envelope is independent of isotopy. A similar result holds for the universal quadratic envelope. We begin with a

Proposition 7. Let $\mu$ be a unital quadratic specialization of $\mathfrak{\Im}$ in $\mathfrak{A}$ and $c$ an invertible element of $\Im$. Then $\mu$ is also a unital quadratic specialization of the isotope $\mathfrak{S}^{(c)}$ in $\mathfrak{A}^{\mu(c)}$. Alternately, the map $\mu^{(c)}(x)=\mu(x) \mu(c)$ defines a unital quadratic specialization of $\mathfrak{S}^{(c)}$ in $\mathfrak{A}$ itself. 
Proof. Recall $\left[4\right.$, p. 1076] that the structure of $\mathfrak{J}^{(c)}$ is given by $1^{(c)}=c^{-1}, U_{x}^{(c)}$ $=U_{x} U_{c}$ and of $\mathfrak{A}^{(d)}$ by $1^{(d)}=d^{-1}, x \cdot{ }_{d} y=x d y$.

One can verify UQS I-III directly, using for UQS III the formulas

$$
\begin{gathered}
\mu\left(x, c^{-1}\right) \mu(c)=\nu(x, c), \quad \mu(c) \mu\left(c^{-1}, x\right)=\nu(c, x), \\
\nu\left(U_{c} y, x\right) \mu(c)=\mu(c) \nu\left(y, U_{c} x\right) .
\end{gathered}
$$

However, a shortcut is available. We can reduce the first case to the second because if $\mu^{(c)}(x)=\mu(x) \mu(c)$ defines a quadratic specialization $\mathfrak{J}^{(c)} \rightarrow \mathfrak{A}$ we can compose it with the (associative) isomorphism $\mathfrak{A} \rightarrow \mathfrak{A}^{\mu(c)}$ given by $a \rightarrow a \mu(c)^{-1}$ to get a quadratic specialization $\mathfrak{\Im}^{(c)} \rightarrow \mathfrak{A}^{\mu(c)}$ by $x \rightarrow \mu(x)$. As for the second case, it suffices to assume $\mu$ is a representation, $\mathfrak{A}=\operatorname{Hom}_{\Phi}(\mathfrak{M}, \mathfrak{M})$; then $c \oplus 0$ is invertible in $\mathfrak{F}=\mathfrak{J} \oplus \mathfrak{M}$, and in the isotope $\mathfrak{F}^{(c)}=\mathfrak{J}^{(c)} \oplus \mathfrak{M}$ we have $U_{x}^{(c)} m=U_{x} U_{c} m=\mu(x) \mu(c) m$, so $\mu^{(c)}(x)=\mu(x) \mu(c)$ defines a representation of $\mathfrak{F}^{(c)}$ on $\mathfrak{M}$ (hence in $\mathfrak{A}$ ).

THEOREM 8. If $\mathfrak{I}$ is a unital quadratic Jordan algebra with universal unital quad-

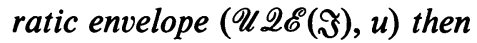

$$
u^{(c)}=u, \quad \mathscr{U} \mathscr{Q} \mathscr{E}\left(\mathfrak{\Im}^{(c)}\right)=\mathscr{U} \mathscr{Q} \mathscr{E}(\Im)^{u(c)},
$$

forms a universal unital quadratic envelope for the isotope $\mathfrak{\Im}^{(c)}, c$ invertible in $\Im$. Alternately we could take

$$
u^{(c)}(x)=u(x) u(c), \quad \mathscr{U} \mathscr{Q} \mathscr{E}\left(\Im^{(c)}\right)=\mathscr{U} \mathscr{Q} \mathscr{E}(\Im) .
$$

Thus the quadratic envelope of the isotope can be obtained either by fixing the universal specialization but modifying the universal algebra, or by fixing the universal algebra but modifying the universal specialization.

Proof. By the proposition, $u^{(c)}$ is a unital quadratic specialization in either case. Also, $u^{(c)}\left(\Im^{(c)}\right)$ generates $\mathscr{U} \mathscr{Q} \mathscr{E}\left(\Im^{(c)}\right)$ in both cases since $u(\Im)$ generates $\mathscr{U} \mathscr{Q} \mathscr{E}(\Im)$. Thus we need only verify that any unital quadratic specialization $\mu: \mathfrak{\Im}^{(c)} \rightarrow \mathfrak{A}$ factors through $u^{(c)}$. We pull $\mu$ back to a specialization $\mu^{(d)}$ of $\mathfrak{J}=\left\{\mathfrak{I}^{(c)}\right\}^{(d)}$ into $\mathfrak{A}$ for $d=c^{-2}$, by the proposition, obtaining a factorization of $\mu^{(d)}$ through $u$ by means of a homomorphism $\hat{\mu}: \mathscr{U} \mathscr{Q} \mathscr{E}(\mathfrak{\Im}) \rightarrow \mathfrak{A}$. We will use this to obtain a factorization of $\mu$ through $u^{(c)}$.

First, if $u^{(c)}(x)=u(x) u(c)$ and $\mathscr{U} \mathscr{Q} \mathscr{E}\left(\mathfrak{\Im}^{(c)}\right)=\mathscr{U} \mathscr{Q} \mathscr{E}(\Im)$ then $\hat{\mu}$ is already a homomorphism $\mathscr{U} \mathscr{Q} \mathscr{E}\left(\mathfrak{\Im}^{(c)}\right) \rightarrow \mathfrak{A}$, and it factors $\mu$ since $\hat{\mu}\left(u^{(c)}(x)\right)=\hat{\mu}(u(x) u(c))=$ $\hat{\mu}(u(x)) \hat{\mu}(u(c))=\mu^{(d)}(x) \mu^{(d)}(c)=\mu(x) \mu(d) \mu(c) \mu(d)=\mu(x) \mu\left(U_{d}^{(c)} c\right)=\mu(x) \mu\left(U_{c}-2 U_{c} c\right)=$ $\mu(x) \mu\left(c^{-1}\right)=\mu(x) \mu\left(1^{(c)}\right)=\mu(x)$ since $\mu$ is a specialization of $\mathfrak{J}^{(c)}$.

If $u^{(c)}=u$ and $\mathscr{U} \mathscr{Q} \mathscr{E}\left(\mathfrak{J}^{(c)}\right)=\mathscr{U} \mathscr{Q} \mathscr{E}(\mathfrak{J})^{u(c)}$ then $\varphi(a)=\hat{\mu}(a) f\left(f=\mu(d)^{-1}\right)$ is a homomorphism of $\mathscr{U} \mathscr{Q} \mathscr{E}\left(\mathfrak{\Im}^{(c)}\right)$ into $\mathfrak{A}$ since $\varphi\left(a \cdot{ }_{u(c)} b\right)=\hat{\mu}(a \cdot u(c) \cdot b) f=\hat{\mu}(a) \hat{\mu}(u(c)) \hat{\mu}(b) f=$ $\hat{\mu}(a) \mu(c) \mu(d) \hat{\mu}(b) f=\hat{\mu}(a) f \mu(d) \mu(c) \mu(d) \hat{\mu}(b) f=\hat{\mu}(a) f \hat{\mu}(b) f=\varphi(a) \varphi(b)$, and it factors $\mu$ since $\varphi\left(u^{(c)}(x)\right)=\hat{\mu}(u(x)) f=\mu(x) \mu(d) f=\mu(x)$.

Thus both $\left.(\mathscr{U} \mathscr{Q} \mathscr{E}(\Im))^{u(c)}, u\right)$ and $(\mathscr{U} \mathscr{Q} \mathscr{E}(\Im), u(x) u(c))$ have the universal property for $\left(\mathscr{U} \mathscr{Q} \mathscr{E}\left(\Im^{(c)}\right), u^{(c)}\right)$.

This formulation is more natural than that for linear Jordan algebras, since the $\rho$ 
corresponding to $u^{(c)}(x)=u(x) u(c)$ is $\rho^{(c)}(x)=\frac{1}{2} \nu^{(c)}(x)=\frac{1}{2} \nu(x, c)=\frac{1}{2}\{\nu(x) \nu(c)-\mu(x, c)\}$ $=2 \rho(x) \rho(c)-\{\rho(x) \rho(c)+\rho(c) \rho(x)-\rho(x \cdot c)\}=[\rho(x), \rho(c)]+\rho(x \cdot c)$ which bears no suggestive relation to the original $\rho$.

5. Compound linear specializations. We can get quadratic specializations from linear ones by composing a linear specialization $\mathfrak{\Im} \stackrel{\sigma}{\rightarrow} \mathfrak{A}$ with the regular quadratic representation $\mathfrak{A} \stackrel{U}{\rightarrow} \operatorname{Hom}_{\Phi}(\mathfrak{A}, \mathfrak{A})$ to obtain a quadratic specialization $\mu(x)=U_{\sigma(x)}$ of $\mathfrak{\Im}$ in $\operatorname{Hom}_{\Phi}(\mathfrak{A}, \mathfrak{A})$.

A more important method of constructing quadratic specializations is to put together two linear ones.

Proposition 9. If $\sigma, \tau$ are commuting unital linear specializations of a unital quadratic Jordan algebra $\mathfrak{\Im}$ in a unital associative algebra $\mathfrak{A}$ then $\mu(x)=\sigma(x) \tau(x)$ $=\tau(x) \sigma(x)$ defines a unital quadratic specialization of $\mathfrak{\Im}$ in $\mathfrak{A}$.

Proof. Clearly UQS I holds if $\sigma, \tau$ are unital: $\mu(1)=\sigma(1) \tau(1)=1$. Also UQS II holds: $\mu\left(U_{x} y\right)=\sigma\left(U_{x} y\right) \tau\left(U_{x} y\right)=\sigma(x) \sigma(y) \sigma(x) \tau(x) \tau(y) \tau(x)=\sigma(x) \tau(x) \sigma(y) \tau(y) \sigma(x) \tau(x)$ $=\mu(x) \mu(y) \mu(x)$ by commutativity of $\sigma(z)$ with $\tau(w)$. To prove USQ III we compute $\nu(x, y)$. Linearizing $\mu$ gives $\mu(x, y)=\sigma(x) \tau(y)+\sigma(y) \tau(x)$, so $\nu(x)=\mu(x, 1)=\sigma(x)+\tau(x)$, and $\nu(x, y)=\nu(x) \nu(y)-\mu(x, y)=\{\sigma(x)+\tau(x)\}\{\sigma(y)+\tau(y)\}-\{\sigma(x) \tau(y)+\sigma(y) \tau(x)\}=$ $\sigma(x) \sigma(y)+\tau(x) \tau(y)$. Thus $\mu\left(U_{x} y, x\right)=\sigma\left(U_{x} y\right) \tau(x)+\sigma(x) \tau\left(U_{x} y\right)=\sigma(x) \sigma(y) \sigma(x) \tau(x)+$ $\sigma(x) \tau(x) \tau(y) \tau(x)$ equals $\mu(x) \nu(y, x)=\sigma(x) \tau(x)\{\sigma(y) \sigma(x)+\tau(y) \tau(x)\}$ and $\nu(x, y) \mu(x)$ $=\{\sigma(x) \sigma(y)+\tau(x) \tau(y)\} \sigma(x) \tau(x)$, proving UQS III. Since $\sigma$ and $\tau$ remain linear specializations in any scalar extension, UQS I-III hold strictly, and $\mu$ is a unital quadratic specialization.

Such a specialization (or representation) $\mu=\sigma \tau$ will be called a unital compound linear specialization (or representation). These have the following important interpretation. We say a unital $\mathfrak{\Im}$-bimodule $\mathfrak{M}$ is special if the split null extension $\mathfrak{E}=\mathfrak{I} \oplus \mathfrak{M}$ is a special Jordan algebra (this of course can only happen when $\mathfrak{\Im}$ itself is special). If $\mathfrak{E} \subset \mathfrak{A}^{+}$for $\mathfrak{A}$ unital associative then the bimodule action of $\mathfrak{\Im}$ on $\mathfrak{M}$ is $\mu_{x}(m)=U_{x} m$ (in the algebra $\mathfrak{E}$ ) $=x m x$ (in the associative algebra $\mathfrak{U}$ ) $=\lambda_{x} \rho_{x}(m)$ where $\lambda_{x}=L_{x}, \rho_{x}=R_{x}$ are left and right multiplications in $\mathfrak{A}$. The restrictions $\sigma, \tau$ of $\lambda, \rho$ to $\mathfrak{\Im}$ are commuting unital linear specializations of $\mathfrak{\Im}$ on $\mathfrak{M}$ with $\mu(x)=\sigma(x) \tau(x)$, so $\mu$ is compound linear. Thus special bimodules are determined by compound linear specializations. Conversely, if $\mathfrak{\Im}$ is special and $\mu=\sigma \tau$ a unital compound linear specialization of $\mathfrak{\Im}$ on $\mathfrak{M}$ then $\mathfrak{\Im} \subset \mathscr{U} \mathscr{L} \mathscr{E}(\mathfrak{\Im})^{+}$and $\sigma$, $\tau$ determine commuting associative homomorphisms $\hat{\sigma}, \hat{\tau}$ of $\mathscr{U} \mathscr{L} \mathscr{E}(\mathfrak{S})$ in $\mathrm{Hom}_{\Phi}(\mathfrak{M}, \mathfrak{M})$. These turn $\mathfrak{M}$ into a unital $\mathscr{U} \mathscr{L} \mathscr{E}(\mathfrak{\Im})$-bimodule by $a m b=\lambda_{a} \rho_{b} m$ for $\lambda_{a}=\hat{\sigma}_{a}, \rho_{b}=\hat{\tau}_{b^{\pi}}$ ( $\pi$ the main involution in $\mathscr{U} \mathscr{L} \mathscr{E}(\Im)$-we must make $\rho$ an antihomomorphism). Thus $\mathfrak{E}=\mathfrak{I} \oplus \mathfrak{M} \subset \mathfrak{A}^{+}$for $\mathfrak{A}=\mathscr{U} \mathscr{L} \mathscr{E}(\mathfrak{\Im}) \oplus \mathfrak{M}$, and compound linear specializations determine special bimodules.

Proposition 10. A unital bimodule $(\mathfrak{M}, \mu)$ for a special unital quadratic Jordan algebra $\mathfrak{\Im}$ is special if and only if the representation $\mu$ is a unital compound linear representation. 
A unital algebra is strongly special if all its unital bimodules are special. It is known [6] that if $\frac{1}{2} \in \Phi$ then any unital Jordan algebra with $n \geqq 4$ interconnected idempotents is special. If $\mathfrak{M}$ is a unital bimodule for such a $\mathfrak{\Im}$ then $\mathfrak{E}=\mathfrak{J} \oplus \mathfrak{M}$ still has the same $n \geqq 4$ interconnected idempotents, so is still special, and therefore $\mathfrak{M}$ is special. In this case any unital $\mathfrak{\Im}$ with $n \geqq 4$ interconnected idempotents is strongly special; for example, this holds for the matrix algebras $\mathfrak{A}_{n}^{+}$or $\mathfrak{S}\left(\mathfrak{A}_{n}, *\right)$ if $\mathfrak{U}$ is associative and $n \geqq 4$. These results no longer hold when $\frac{1}{2} \notin \Phi$, as we shall see shortly. It is true that an $n$-interconnected Jordan algebra with zero extreme radical is special if $n \geqq 4$, so if $\Im$ has zero extreme radical and so does its bimodule $\mathfrak{M}$ (in the sense that $\mu(\mathfrak{F}, \mathfrak{\Im}) m=0$ implies $m=0$ ) then $\mathfrak{E}=\mathfrak{I} \oplus \mathfrak{M}$ will have no extreme radical and hence be special. Thus the "nonpathological" bimodules for such algebras as $\mathfrak{A}_{n}^{+}$or $\mathfrak{S}\left(\mathfrak{A}_{n}, *\right)$ will be compound linear, even though the algebras may not be strongly special in general.

We can form a universal object for such compound linear specializations. A universal unital compound linear envelope for $\mathfrak{\Im}$ consists of a unital associative algebra $\mathscr{U} \mathscr{C} \mathscr{E}(\Im)$ together with a universal unital compound linear specialization $c$ of $\mathfrak{\Im}$ in $\mathscr{U} \mathscr{C} \mathscr{E}(\Im)$ such that any unital compound linear specialization $\mu=\sigma \tau$ factors uniquely through $c$ :

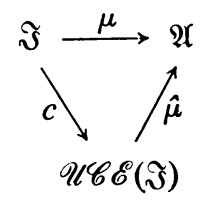

To construct $\mathscr{U} \mathscr{C} \mathscr{E}(\Im)$, note that if $\mu=\sigma \tau$ the commuting specializations $\sigma, \tau$ extend to commuting associative homomorphisms $\hat{\sigma}, \hat{\tau}: \mathscr{U} \mathscr{L} \mathscr{E}(\mathfrak{\Im}) \rightarrow \mathfrak{A}$ (as before), so we have a unital homomorphism $\hat{\mu}=\hat{\sigma} \otimes \hat{\tau}: \mathscr{U} \mathscr{L} \mathscr{E}(\Im) \otimes \mathscr{U} \mathscr{L} \mathscr{E}(\Im) \rightarrow \mathfrak{A}$ and a

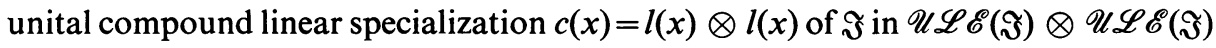
(for $l: \mathfrak{\Im} \rightarrow \mathscr{U} \mathscr{L} \mathscr{E}(\Im)$ the universal unital linear specialization) such that $\hat{\mu} \circ c=\mu$ since $\hat{\mu}(c(x))=\hat{\mu}(l(x) \otimes l(x))=\hat{\sigma}(l(x)) \hat{\tau}(l(x))=\sigma(x) \tau(x)=\mu(x)$. However, this factorization need not be unique since $c(\Im)$ need not generate all of $\mathscr{U} \mathscr{L} \mathscr{E}(\Im)$ $\otimes \mathscr{U} \mathscr{L} \mathscr{E}(\Im)$. We obtain

Proposition 11. If $\mathscr{U} \mathscr{C} \mathscr{E}(\Im)$ denotes the subalgebra of $\mathscr{U} \mathscr{L} \mathscr{E}(\Im) \otimes \mathscr{U} \mathscr{L} \mathscr{E}(\Im)$ generated by all $c(x)=l(x) \otimes l(x)$ then $\mathscr{U} \mathscr{C} \mathscr{E}(\Im)$ and $c$ form a universal unital compound linear envelope for $\Im$.

Since $c$ is in particular a unital quadratic specialization, it factors through the universal unital quadratic specialization:

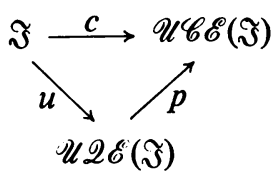


for $p$ the canonical projection of $\mathscr{U} \mathscr{Q} \mathscr{E}$ onto $\mathscr{U} \mathscr{C} \mathscr{E}$ (it is surjective since its range contains the generators $c(x)=p(u(x)))$.

Proposition 12. $\Im$ is strongly special if and only if the canonical projection $p$ is an isomorphism of $\mathscr{U} \mathscr{Q} \mathscr{E}(\Im)$ onto $\mathscr{U} \mathscr{C} \mathscr{E}(\Im)$.

Proof. If $p$ is an isomorphism then any unital quadratic specialization is compound linear since it factors through $c$ ( $c$ and $\mathscr{U} \mathscr{C} \mathscr{E}(\Im)$ serve as universal unital quadratic envelope), so by Proposition $10, \Im$ is strongly special. Conversely, if all quadratic specializations are compound linear they factor through $c$, hence $c$ and $\mathscr{U} \mathscr{C} \mathscr{E}(\Im)$ serve as universal unital quadratic envelope, so by uniqueness $p$ must be an isomorphism.

Compound linear specializations also come up in considering quadratic specializations of direct sums. Suppose $\sigma_{i}: \mathfrak{\Im}_{i} \rightarrow \mathfrak{A}$ are commuting unital linear specializations $(i=1,2)$. Then $\tau_{i}: \mathfrak{\pi _ { i }} \rightarrow \mathfrak{\Im}_{i} \stackrel{\sigma_{\mathfrak{i}}}{\rightarrow} \mathfrak{A}$ are also commuting unital linear specializations of the direct sum $\mathfrak{I}=\mathfrak{I}_{1} \oplus \Im_{2}$. From these we construct a compound linear specialization having $\mu(x)=\mu\left(x_{1} \oplus x_{2}\right)=\sigma_{1}\left(x_{1}\right) \sigma_{2}\left(x_{2}\right)$ and $\nu\left(x_{1} \oplus x_{2}\right)$ $=\sigma_{1}\left(x_{1}\right)+\sigma_{2}\left(x_{2}\right)$. The universal gadget for such specializations consists of the algebra $\mathscr{U} \mathscr{C} \mathscr{E}\left(\Im_{1}, \Im_{2}\right)=\mathscr{U} \mathscr{L} \mathscr{E}\left(\Im_{1}\right) \otimes \mathscr{U} \mathscr{L} \mathscr{E}\left(\mathfrak{\Im}_{2}\right)$ together with the unital quadratic specialization $c(x)=c\left(x_{1} \oplus x_{2}\right)=l_{1}\left(x_{1}\right) \otimes l_{2}\left(x_{2}\right)$ of $\Im=\Im_{1} \oplus \Im_{2}$ in $\mathscr{U} \mathscr{C} \mathscr{E}\left(\Im_{1}, \Im_{2}\right)$; it has the universal property that any compound linear specialization $\mu(x)=\sigma_{1}\left(x_{1}\right) \sigma_{2}\left(x_{2}\right)$ of $\mathfrak{\Im}_{1} \oplus \mathfrak{\Im}_{2}$ in $\mathfrak{A}$ factors uniquely through $c$ by means of an associative homomorphism $\mathscr{U} \mathscr{C} \mathscr{E}\left(\Im_{1}, \mathfrak{\Im}_{2}\right) \rightarrow \mathfrak{A}$ (note that in this case the $c\left(x_{1} \oplus x_{2}\right)$ do generate all of $\left.\mathscr{U} \mathscr{L} \mathscr{E}\left(\Im_{1}\right) \otimes \mathscr{U} \mathscr{L} \mathscr{E}\left(\Im_{2}\right)\right)$.

The specializations obtained in this way have $\mu\left(\mathfrak{I}_{1}\right)=\mu\left(\mathfrak{\Im}_{2}\right)=0$, or equivalently $\mu\left(e_{1}, e_{2}\right)=1$ for $e_{i}$ the unit of $\Im_{i}$. We will now see that any $\mu: \mathfrak{I}_{1} \oplus \mathfrak{I}_{2} \rightarrow \mathfrak{A}$ having this property is obtained by this process.

Proposition 13. If $\mathfrak{\Im}_{1}, \mathfrak{\Im}_{2}$ are unital quadratic Jordan algebras then the universal unital quadratic envelope and universal unital quadratic specialization for $\mathfrak{\Im}=\Im_{1} \oplus \mathfrak{I}_{2}$ factor into direct sums

$$
\mathscr{U} \mathscr{L} \mathscr{E}(\Im)=\mathfrak{Q}_{11} \oplus \mathfrak{Q}_{12} \oplus \mathfrak{Q}_{22} \quad u=u_{11} \oplus u_{12} \oplus u_{22}
$$

where the ideals $\mathfrak{Q}_{i i}$ and unital quadratic specializations $u_{i i}$ form universal unital quadratic envelopes for $\Im_{i}$ and the ideal $\mathfrak{Q}_{12}$ together with $u_{12}$ forms a universal unital compound linear envelope for $\left(\Im_{1}, \mathfrak{\Im}_{2}\right)$ :

$$
\mathscr{U} \mathscr{Q} \mathscr{E}\left(\Im_{1} \oplus \Im_{2}\right)=\mathscr{U} \mathscr{Q} \mathscr{E}\left(\Im_{1}\right) \oplus \mathscr{U} \mathscr{E} \mathscr{E}\left(\Im_{1}, \mathfrak{I}_{2}\right) \oplus \mathscr{U} \mathscr{Q} \mathscr{E}\left(\Im_{2}\right)
$$

Proof. From $1=e_{1}+e_{2}$ in $\Im\left(e_{i}\right.$ the unit of $\left.\Im_{i}\right)$ we get $1=u(1)=u\left(e_{1}\right)+u\left(e_{1}, e_{2}\right)$ $+u\left(e_{2}\right)=e_{11}+e_{12}+e_{22}$ in $\mathscr{U} \mathscr{Q} \mathscr{E}(\Im)$. The $e_{i i}$ are idempotents since $u\left(e_{i}\right)^{2}=u\left(e_{i}^{2}\right)$ $=u\left(e_{i}\right)$ by UQS I, II, and they are orthogonal since $e_{i i} e_{j j}=u\left(e_{i}\right) u\left(1-e_{i}\right)=$ $u\left(e_{i}\right)\left\{1-v\left(e_{i}\right)+u\left(e_{i}\right)\right\}=u\left(e_{i}\right)-u\left(e_{i}^{2}, e_{i}\right)+u\left(e_{i}\right)$ (by UQS III with $\left.y=1\right)=0$. Hence also $e_{12}=1-e_{11}-e_{22}$ is an idempotent orthogonal to all the others. We claim that in the Peirce decomposition of $\mathfrak{Q}=\mathscr{U} \mathscr{Q} \mathscr{E}(\Im)$ only the spaces $\mathfrak{Q}_{i j}=e_{i j} \mathfrak{Q} e_{i j}$ appear. To 
see this, note first that $\mathfrak{Q}$ is generated by $u(\mathfrak{\Im})=u\left(\Im_{1}\right)+u\left(\Im_{1}, \Im_{2}\right)+u\left(\Im_{2}\right)$ where the $u\left(\Im_{i}\right)=u\left(U_{e_{i}} \mathfrak{\Im}\right)=u\left(e_{i}\right) u(\Im) u\left(e_{i}\right)$ belong to $e_{i i} \mathfrak{Q} e_{i i}=\mathfrak{Q}_{i i}$. If we can show that $u\left(\mathfrak{I}_{1}, \mathfrak{I}_{2}\right) \subset \mathfrak{Q}_{12}$ then the generators all belong to $\mathfrak{Q}_{11}+\mathfrak{Q}_{12}+\mathfrak{Q}_{22}$, and $\mathfrak{Q}_{=}=\mathfrak{Q}_{11}$ $\oplus \mathfrak{Q}_{12} \oplus \mathfrak{Q}_{22}$ is a direct sum of ideals. Now $u\left(\mathfrak{\Im}_{1}, \mathfrak{\Im}_{2}\right)=u\left(U_{e_{1}} \mathfrak{\Im}_{1}, \mathfrak{\Im}_{2}\right)+u\left(U_{e_{1}} \mathfrak{\Im}_{2}, \mathfrak{\Im}_{1}\right)$ (since $U_{e_{1}}=I$ on $\mathfrak{I}_{1}$ and $=0$ on $\left.\mathfrak{\Im}_{2}\right)=v\left(e_{1}, \Im_{1}\right) u\left(e_{1}, \mathfrak{I}_{2}\right)-u\left(e_{1}\right) v\left(\Im_{1}, \mathfrak{\Im}_{2}\right)$ (by $(10)$ ) $=v\left(e_{1}\right) v\left(\Im_{1}\right) u\left(e_{1}, \Im_{2}\right)-u\left(e_{1}, \Im_{1}\right) u\left(e_{1}, \Im_{2}\right)-u\left(e_{1}\right) v\left(\Im_{1}, \Im_{2}\right) \subset e_{12} \mathfrak{D}+e_{11} \mathfrak{Q}$ since $v\left(e_{1}\right)$ $=u\left(e_{1}, e_{1}+e_{2}\right)=2 e_{11}+e_{12}$. Similarly $u\left(\Im_{1}, \Im_{2}\right) \subset e_{12} \mathfrak{Q}+e_{22} \mathfrak{Q}$, so $u\left(\Im_{1}, \Im_{2}\right) \subset e_{12} \mathfrak{D}$. Dually $u\left(\Im_{1}, \mathfrak{\Im}_{2}\right) \subset \mathfrak{D} e_{12}$.

We have a corresponding decomposition $u=u_{11} \oplus u_{12} \oplus u_{22}$. We claim $\left(\mathfrak{Q}_{i i}, u_{i i}\right)$ has the universal property for the universal unital quadratic envelope for $\Im_{i}$. We know $u_{i i}$ is a unital quadratic specialization of $\Im_{i}$ in $\mathfrak{Q}_{i i}$ since $u\left(e_{i}\right)=e_{i i}$, and $u_{i i}\left(\Im_{i}\right)=u\left(\Im_{i}\right)=u_{i i}(\Im)$ generates $\mathfrak{Q}_{i i}$. Furthermore, any unital quadratic specialization $\mu_{i}: \Im_{i} \rightarrow \mathfrak{A}$ extends to a unital quadratic specialization $\mu: \mathfrak{\Im} \stackrel{\pi_{i}}{\rightarrow} \mathfrak{I}_{i} \stackrel{\mu_{i}}{\rightarrow} \mathfrak{A}$, so $\mu=\hat{\mu} \circ \boldsymbol{u}$ for some associative homomorphism $\hat{\mu}: \mathfrak{Q} \rightarrow \mathfrak{A}$. If $\hat{\mu}_{i i}$ denotes the restriction of $\hat{\mu}$ to $\mathfrak{Q}_{i i}$ then $\hat{\mu}_{i i} \circ u_{i i}=\mu_{i}$ as desired.

It is easy to see that any unital compound linear specialization $\mu: \Im_{1} \oplus \mathfrak{I}_{2} \rightarrow \mathfrak{A}$ factors uniquely through $u_{12}$ by means of an associative homomorphism $\hat{\mu}: \mathfrak{Q}_{12} \rightarrow \mathfrak{A}$ ( $\mu$ factors through $u$ and $\hat{\mu}: \mathfrak{Q} \rightarrow \mathfrak{A}$, but since $\mu\left(\Im_{1}\right)=\mu\left(\Im_{2}\right)=0$ we have $\hat{\mu}\left(\mathfrak{Q}_{11}\right)$ $=\hat{\mu}\left(\mathfrak{Q}_{22}\right)=0$ and $\hat{\mu}$ restricts to $\left.\mathfrak{Q}_{12}\right)$. We must check that $u_{12}$ is actually a compound linear specialization. We have

$$
u_{12}\left(x_{1} \oplus x_{2}\right)=u_{12}\left(x_{1}, x_{2}\right)=v_{1}\left(x_{1}\right) v_{2}\left(x_{2}\right)=v_{2}\left(x_{2}\right) v_{1}\left(x_{1}\right)
$$

where the $v_{i}$ are the restrictions to $\Im_{i}$ of the $\mathfrak{Q}_{12}$ component $e_{12} v e_{12}$ of $v$. Indeed, $v\left(x_{1}\right) v\left(x_{2}\right)-u\left(x_{1}, x_{2}\right)=v\left(x_{1}, x_{2}\right)=v\left(U_{e_{1}} x_{1}, x_{2}\right)+v\left(U_{e_{1}} x_{2}, x_{1}\right)=v\left(e_{1}, U_{x_{1}, x_{2}} e_{1}\right)=0$ by orthogonality and linearized (9), so taking components in $\mathfrak{Q}_{12}$ gives $v_{1}\left(x_{1}\right) v_{2}\left(x_{2}\right)$ $-u_{12}\left(x_{1}, x_{2}\right)=0$. The $v_{i}$ are unital linear specializations since

$$
v_{i}\left(e_{i}\right)=e_{12}
$$

by $v\left(e_{i}\right)=2 e_{i i}+e_{12}$, and

$$
v_{i}\left(U_{x_{i}} y_{i}\right)=v_{i}\left(x_{i}\right) v_{i}\left(y_{i}\right) v_{i}\left(x_{i}\right)
$$

by taking $\mathfrak{Q}_{12}$ components of $v\left(U_{x_{i}} y_{i}\right)=v\left(x_{i}\right) v\left(y_{i}\right) v\left(x_{i}\right)-v\left(x_{i}\right) u\left(x_{i}, y_{i}\right)-v\left(y_{i}\right) u\left(x_{i}\right)$ (as in [5, Lemma $1,(2.11)]$ or (19) below).

COROLlaRY 14. If $\Im_{1}, \Im_{2}$ are unital quadratic Jordan algebras then any unital quadratic specializations $\mu$ of $\Im_{1} \oplus \Im_{2}$ is the direct sum of a unital quadratic specialization $\mu_{11}$ of $\Im_{1}$, a unital quadratic specialization $\mu_{22}$ of $\Im_{2}$, and a unital compound linear specialization $\mu_{12}$ of $\left(\Im_{1}, \Im_{2}\right)$.

As a final remark on compound linear specializations, note that if $\frac{1}{2} \in \Phi$ then $\mu(x)=\sigma(x) \tau(x), \nu(x)=\sigma(x)+\tau(x)$ is associated with the representation $\rho(x)=\frac{1}{2} \nu(x)$ $=\frac{1}{2}\{\sigma(x)+\tau(x)\}$ of the linear Jordan algebra determined by $\Im$. Thus $\rho$ is the "average" of $\sigma$ and $\tau$. Note, however, that this average $\rho$ can only be defined when $\frac{1}{2} \in \Phi$, whereas we can always define $\mu$ and $\nu$. 
6. Pathologies. In certain characteristic 2 situations we obtain a new type of quadratic specialization having no analogue in characteristic $\neq 2$. Consider a unital quadratic specialization $\mu: \mathfrak{\Im} \rightarrow \mathfrak{A}$ such that

$$
\mu(x, y) \equiv 0
$$

for all $x, y$. Then $2 \mathfrak{A}=\mu(1,1) \mathfrak{A}=0$ (so if $\frac{1}{2} \in \Phi, \mathfrak{A}=0$ and $\mu$ is trivial). We are going to turn $\mu$ into a unital linear specialization $\sigma: \mathfrak{I} \rightarrow \tilde{\mathfrak{A}}$. We let $\tilde{\mathfrak{A}}$ be $\mathfrak{A}$ as a ring,

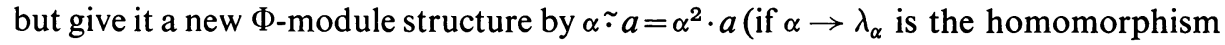
of $\Phi$ into the centroid $\Gamma(\mathfrak{A})$ turning $\mathfrak{A}$ into a $\Phi$-algebra, the new homomorphism is $\tilde{\lambda}_{\alpha}=\lambda_{\alpha^{2}}$; clearly $\tilde{\lambda}_{1}=\lambda_{1}=1, \quad \tilde{\lambda}_{\alpha} \tilde{\lambda}_{\beta}=\tilde{\lambda}_{\alpha \beta}, \quad \tilde{\lambda}_{\alpha+\beta}=\lambda_{(\alpha+\beta)}{ }^{2}=\lambda_{\alpha^{2}+2 \alpha \beta+\beta^{2}}=\tilde{\lambda}_{\alpha}+\tilde{\lambda}_{\beta}$ since $2 \mathfrak{A}=0)$. Then $\sigma=\mu: \mathfrak{\Im} \rightarrow \mathfrak{A}$ is a unital linear specialization because $\sigma(1)=\mu(1)=1$, $\sigma\left(U_{x} y\right)=\mu\left(U_{x} y\right)=\mu(x) \mu(y) \mu(x)=\sigma(x) \sigma(y) \sigma(x), \sigma(x+y)=\mu(x+y)=\mu(x)+\mu(x, y)$ $+\mu(y)=\mu(x)+\mu(y)=\sigma(x)+\sigma(y) \quad$ (by hypothesis), and $\sigma(\alpha x)=\mu(\alpha x)=\alpha^{2} \cdot \mu(x)$ $=\alpha \sim \sigma(x)$. The resulting algebra $\tilde{\mathfrak{A}}$ is a $\sqrt{ } \Phi$-algebra in the sense that the module structure $\tilde{\lambda}: \Phi \rightarrow \Gamma(\tilde{\mathfrak{A}})$ has $\tilde{\lambda}=\lambda^{2}$ for some homomorphism $\lambda: \Phi \rightarrow \Gamma(\tilde{\mathfrak{A}})$. We say $\sigma$ is obtained from $\mu$ by deflation (from quadratic to linear).

Conversely, suppose $\sigma: \mathfrak{\Im} \rightarrow \tilde{\mathfrak{A}}$ is a unital linear specialization of $\mathfrak{\Im}$ in a $\sqrt{ } \Phi$ algebra $\tilde{\mathfrak{A}}$. We obtain a unital quadratic specialization $\mu: \mathfrak{\Im} \rightarrow \mathfrak{A}$ by the reverse process: we set $\mu(x)=\sigma(x)$ and give $\mathfrak{A}$ the same ring structure as $\tilde{\mathfrak{A}}$ but the new module structure determined by $\lambda$ (where $\tilde{\lambda}=\lambda^{2}$ ). Then we have $\mu(1)=\sigma(1)=1$, $\mu\left(U_{x} y\right)=\sigma\left(U_{x} y\right)=\sigma(x) \sigma(y) \sigma(x)=\mu(x) \mu(y) \mu(x), \mu\left(U_{x} y, x\right)=\nu(x, y) \mu(x)=\mu(x) \nu(y, x)$ $=0$ since $\mu(x, y)=\sigma(x+y)-\sigma(x)-\sigma(y)=0$. The map $\mu$ is quadratic by the way we changed the module structure: $\mu(x, y)=0$ is trivially bilinear, and $\mu(\alpha x)=\sigma(\alpha x)$ $=\alpha \tilde{\tau} \sigma(x)=\alpha^{2} \cdot \mu(x)$. We say $\mu$ is obtained from $\sigma$ by inflation.

Thus there is a natural 1-1 correspondence between unital quadratic specializations with $\mu(x, y) \equiv 0$ and unital linear specializations in $\sqrt{ } \Phi$-algebras; the $\mu$ with $\mu(x, y) \equiv 0$ are precisely the inflated linear specializations.

Such inflated linear specializations are common when $\Phi$ is a field of characteristic 2. Let $\sigma: \mathfrak{\Im} \rightarrow \tilde{\mathfrak{A}}$ be any unital linear specialization; we enlarge $\tilde{\mathfrak{A}}$ slightly to obtain a specialization $\mathfrak{\Im} \rightarrow \tilde{\mathfrak{A}}_{\Omega}=\Omega \otimes_{\Phi} \tilde{\mathfrak{A}}$ for $\Omega$ a perfect closure of $\Phi$ (e.g. if $\Phi$ is finite or algebraically closed we can take $\Omega=\Phi)$. The map $\omega \rightarrow \sqrt{ } \omega$ is an automorphism of $\Omega$ since $\Omega$ is perfect of characteristic 2, so the natural $\Omega$-module structure $\tilde{\lambda}$ of $\tilde{\mathfrak{A}}_{\Omega}$ has $\tilde{\lambda}=\lambda^{2}$ for $\lambda_{\omega}=\tilde{\lambda}_{\sqrt{ } \omega}$. Thus $\tilde{\mathfrak{A}}_{\Omega}$ is a $\sqrt{ } \Phi$ (even a $\sqrt{ } \Omega$ ) algebra. (Note $\tilde{\mathfrak{A}}$ is not invariant under $\lambda_{\alpha}$ for $\alpha \in \Phi$ in general, so we have to pass outside $\tilde{\mathfrak{A}}$ to $\tilde{\mathfrak{A}}_{\Omega}$.) This shows that any unital linear specialization over a field $\Phi$ of characteristic 2 can be enlarged to a specialization in a $\sqrt{ } \Phi$-algebra, hence gives rise to an inflated linear specialization.

These inflated linear specializations are seldom compound linear. If $\mu(x, y) \equiv 0$ and $\mu(x)=\sigma(x) \tau(x)$ then $\sigma(x)+\tau(x)=\mu(x, 1)=0$ and $2=\mu(1,1)=0$ show that $\sigma(x)=\tau(x)$ and all $\sigma(x), \sigma(y)$ commute; we may assume $\sigma(\Im)$ and $\tau(\mathfrak{\Im})$ generate $\mathfrak{A}$, hence that $\mathfrak{A}$ is commutative. Besides this restriction on $\mathfrak{A}, \mathfrak{I}$ is severely restricted: the kernel of $\sigma$ is an ideal containing $\Im \circ \Im$ since $\sigma(x \circ y)=\sigma(x) \circ \sigma(y)=0$ when $\mathfrak{A}$ 
is commutative of characteristic 2 . If $\mathfrak{\Im}$ is simple but $\mathfrak{\Im} \circ \mathfrak{\Im} \neq 0$, or if $\mathfrak{\Im}$ has $n \geqq 2$ interconnected idempotents, the only ideal containing $\mathfrak{\Im} \Im$ is $\Im$ itself (if $e_{i}$ are idempotents such a $\sigma$ would kill the Peirce spaces $\Im_{i j}=e_{i} \circ \Im_{i j}$ for $i \neq j$, hence also the $\mathfrak{\Im}_{i i}=U_{\Im_{i}} \Im_{j j}$ if $e_{i}$ and $e_{j}$ are interconnected, so it would kill $\mathfrak{\Im}=\oplus \mathfrak{\Im}_{i j}$ ), so the only such $\sigma$ is $\sigma \equiv 0$. Thus as mentioned before the matrix algebras $\mathfrak{A}_{n}^{+}$and $\mathfrak{S}\left(\mathfrak{U}_{n}, *\right)$ in characteristic 2 have quadratic specializations (the inflated linear ones) which are not compound linear, and hence the algebras are not strongly special.

The exceptional algebra is an exception to this pathology-since $\mathfrak{S}_{(}\left(\mathfrak{C}_{3}\right)$ has no nonzero linear specializations at all it has no nonzero inflated linear specializations.

If $\mathfrak{\Im}$ is a unital quadratic Jordan algebra its extreme radical or kernel $\mathbf{Z}$ furnishes an inflated linear specialization: 8 is an ideal, hence a bimodule relative to the

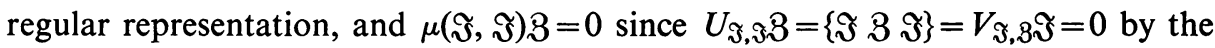
definition $U_{8}=U_{8, \Im}=0$ of the kernel.

\section{The General CASE}

A quadratic Jordan algebra is a triple $\mathfrak{\Im}=(\mathfrak{X}, U, 2)$ where $x \rightarrow U_{x}$ and $x \rightarrow x^{2}$ are quadratic maps of $\mathfrak{X}$ into $\operatorname{Hom}_{\Phi}(\mathfrak{X}, \mathfrak{X})$ and $\mathfrak{X}$ respectively, such that the following axioms hold strictly:

(QJ I) $V_{x, x}=V_{x^{2}}$,

(QJ II) $U_{x} V_{x}=V_{x} U_{x}$,

(QJ III) $U_{x}\left(x^{2}\right)=\left(x^{2}\right)^{2}$,

(QJ IV) $U_{x} U_{y}\left(x^{2}\right)=\left(U_{x} y\right)^{2}$,

(QJ V) $U_{x^{2}}=U_{x}^{2}$

(QJ VI) $U_{U(x) y}=U_{x} U_{y} U_{x}$,

where, as usual, $V_{x} y=x \circ y=(x+y)^{2}-x^{2}-y^{2}$ and $V_{x, y} z=\left\{\begin{array}{lll}x & z & z\end{array}\right\}=U_{x, z} y=$ $\left\{U_{x+z}-U_{x}-U_{z}\right\} y$.

A homomorphism $\varphi: \mathfrak{\Im} \rightarrow \widetilde{\mathfrak{\Im}}$ satisfies $\varphi\left(x^{2}\right)=\varphi(x)^{\tilde{2}}$ and $\varphi\left(U_{x} y\right)=\widetilde{U}_{\varphi(x)} \varphi(y)$. In the resulting category a subalgebra is a subspace $\mathfrak{N}$ of $\mathfrak{\Im}$ such that $\mathfrak{\pi}^{2} \subset \mathfrak{\Re}$ and $U_{\Re} \mathfrak{T} \subset \mathfrak{\Re}$, while an ideal satisfies $\mathfrak{\Re}^{2} \subset \Re, \mathfrak{R} \circ \mathfrak{\Im} \subset \Re, U_{\Re} \mathfrak{A}, U_{\mathfrak{S}} \mathfrak{A}$. A unit in a quadratic Jordan algebra is an element $c$ such that $U_{c}=I$ and $U_{x} c=x^{2}$ for all $x$. Quadratic Jordan algebras with unit are equivalent to unital quadratic Jordan algebras.

If a quadratic Jordan algebra $\mathfrak{\Im}$ does not contain a unit we can always imbed it in the canonical unital extension $\mathfrak{J}^{\prime}=\Phi 1+\mathfrak{\Im}$ which is a unital quadratic Jordan algebra. We use this (and an analogous result for specializations) to reduce questions about general Jordan algebras to the unital case. (For these results about quadratic Jordan algebras see [5].)

1. Representation theory. If $X$ is any subset of a quadratic Jordan algebra $\mathfrak{F}$ the multiplication algebra $\mathscr{M}_{\Im}(X)$ determined by $X$ is the algebra of transformations on $\mathfrak{\Im}$ generated by all $V_{x}, U_{x}, U_{x, y}$ for $x, y$ in $X$. We again have a multiplication closure theorem

$$
\mathscr{M}\left(\mathscr{M}^{\prime}(X) X\right)=\mathscr{M}(X)
$$


and its corollaries that the subalgebra generated by $X$ is

$$
\Phi[X]=\mathscr{M}^{\prime}(X) X
$$

and that if $X$ generates $\Re$ then the $V_{x}, U_{x}, U_{x, y}$ generate $\mathscr{M}(\Re)$. (We have to use the unital algebra $\mathscr{M}^{\prime}(X)=\Phi I+\mathscr{M}(X)$ since $\mathscr{M}(X)$ may not contain $I$ and hence $\mathscr{M}(X) X$ may not contain $X$.) All these follow because $X^{\prime}=X \cup\{1\}$ is a unital subset of $\mathfrak{J}^{\prime}=\Phi 1+\mathfrak{J}$ and $\mathscr{M}_{\Im^{\prime}}\left(X^{\prime} \mid \mathfrak{\Im}\right)=\mathscr{M}^{\prime}(X)$ since the algebra generated by the $U_{x^{\prime}}, U_{x^{\prime}, y^{\prime}}$ coincides with the algebra generated by $U_{1}=I$ and all $U_{x}, U_{x, y}, U_{x, 1}=V_{x}$. Mappings of the form $x \rightarrow U_{x}$ of $\mathfrak{I}$ into $\mathscr{M}_{\mathfrak{E}}(\mathfrak{I} \mid \mathfrak{I})$ for $\mathfrak{\Im}$ a subalgebra of $\mathfrak{E}$ and $\mathfrak{\Omega}$ a subspace of $\&$ invariant under $\mathfrak{\Im}$ are called multiplication representations of $\mathfrak{~}$.

A quadratic specialization of $\mathfrak{I}$ in an associative algebra $\mathfrak{A}$ is a pair $(\mu, \nu)$ of maps from $\mathfrak{\Im}$ to $\mathfrak{A}$, where $\mu$ is quadratic and $\nu$ linear, such that the following axioms hold strictly:

(QS I) $v(x, x)=v\left(x^{2}\right)$,

(QS II) $\mu\left(x^{2}, x\right)=\mu(x) \nu(x)=\nu(x) \mu(x)$,

(QS III) $\mu(x \circ y, x)+\nu\left(U_{x} y\right)=\nu(x) \nu(y, x)+\mu(x) \nu(y)=\nu(x, y) \nu(x)+\nu(y) \mu(x)$,

(QS IV) $\mu\left(U_{x} y, x\right)=\mu(x) \nu(y, x)=\nu(x, y) \mu(x)$,

(QS V) $\mu\left(x^{2}\right)=\mu(x)^{2}$,

(QS VI) $\mu\left(U_{x} y\right)=\mu(x) \mu(y) \mu(x)$,

where $\nu(x, y)=\nu(x) \nu(y)-\mu(x, y)$ by definition. Note that (QS I) is thus equivalent to

(QS I') $2 \mu(x)=\nu(x)^{2}-\nu\left(x^{2}\right)$.

A quadratic representation of $\mathfrak{\Im}$ on $\mathfrak{M}$ is a quadratic specialization of $\mathfrak{I}$ in $\mathfrak{A}=\operatorname{Hom}_{\Phi}(\mathfrak{M}, \mathfrak{M})$, and a bimodule for $\mathfrak{\Im}$ is a space $\mathfrak{M}$ together with a quadratic representation of $\mathfrak{I}$ on $\mathfrak{M}$.

If $\Im$ is a unital quadratic Jordan algebra and $\mu$ a unital quadratic specialization of $\Im$, then $\mu$ and $\nu(x)=\mu(x, 1)$ satisfy the axioms QS I-VI: QS VI is UQS II, QS V results from UQS II by setting $y=1$, QS IV is UQS III, QS III results by linearizing $x \rightarrow x, 1$ in UQS III, QS II follows by setting $y=1$ in UQS III, while QS I' results by linearizing $x \rightarrow x, 1$ in QS V. Clearly if QS I-VI hold for an algebra they hold for any subalgebra. Thus if $\mathfrak{\Im}$ is a subalgebra of a unital algebra $\mathfrak{J}^{\prime}$, any unital specialization $\mu^{\prime}$ of $\Im^{\prime}$ restricts to a specialization of $\Im$. We shall soon see that all specializations of $\mathfrak{I}$ arise in this manner.

We can get an alternate axiomatization based on the compositions $x^{2}$ and $x^{3}$ rather than $U_{x} y$.

Proposition 15. $(\mu, v)$ is a quadratic specialization if and only if

(CS I) $\nu\left(x^{2}\right)=\nu(x, x)$,

(CS II) $\nu\left(x^{3}\right)=\nu\left(x, x^{2}\right)=\nu\left(x^{2}, x\right)$,

(CS III) $\mu\left(x^{2}, y\right)=\mu(x, y) \nu(x)-\nu(y) \mu(x)=\nu(x) \mu(x, y)-\mu(x) \nu(y)$,

(CS IV) $\mu\left(x^{3}, y\right)=\mu(x, y) \nu\left(x^{2}\right)-\nu(y, x) \mu(x)=\nu\left(x^{2}\right) \mu(y, x)-\mu(x) \nu(x, y)$,

(CS V) $\mu\left(x^{2}\right)=\mu(x)^{2}$,

(CS VI) $\mu\left(x^{3}\right)=\mu(x)^{3}$

hold strictly. 
The proof consists in juggling identities as in [5]. For our present purposes we only show that QS I-VI imply a strengthening of QS III:

$$
\begin{gathered}
\nu\left(U_{x} y\right)=\nu(x) \nu(y, x)-\nu(y) \mu(x)=\nu(x, y) \nu(x)-\mu(x) \nu(y), \\
\mu(x \circ y, x)=\mu(x) \nu(y)+\nu(y) \mu(x) .
\end{gathered}
$$

By QS III, (19) and (20) are equivalent, and by linearizing QS II we see (20) is equivalent to

$$
\mu\left(x^{2}, y\right)=\mu(x, y) \nu(x)-\nu(y) \mu(x)=\nu(x) \mu(x, y)-\mu(x) \nu(y) .
$$

Setting $y=x$ in QS III gives $\nu\left(x^{3}\right)+2 \mu\left(x^{2}, x\right)=\nu(x) \nu\left(x^{2}\right)+\mu(x) \nu(x)$ (by QS I), hence $\nu\left(x^{3}\right)+\mu\left(x^{2}, x\right)=\nu(x) \nu\left(x^{2}\right)$ (by QS II), and linearizing this gives $0=\nu\left(U_{x} y\right)+\nu\left(x^{2} \circ y\right)$ $+\mu(x \circ y, x)+\mu\left(x^{2}, y\right)-\nu(y) \nu\left(x^{2}\right)-\nu(x) \nu(x \circ y)=\{\nu(x) \nu(y, x)+\mu(x) \nu(y)\}+\left\{\nu\left(x^{2}\right) \nu(y)\right.$ $\left.-\mu\left(x^{2}, y\right)\right\}-\nu(x)\{\nu(x, y)+\nu(y, x)\}$ (by QS III, linearized QS $\mathrm{I}^{\prime}$, linearized QS I) $=\left\{\nu(x)^{2}-\mu(x)\right\} \nu(y)-\mu\left(x^{2}, y\right)-\nu(x) \nu(x, y) \quad$ (by $\quad$ QS $\left.\quad \mathrm{I}^{\prime}\right)=\{\nu(x) \mu(x, y)-\mu(x) \nu(y)\}$ $-\mu\left(x^{2}, y\right)$, establishing (21).

Just as any Jordan algebra is contained in a unital one, any quadratic specialization is contained in a unital one.

THEOREM 16 (ADJUNCTION THEOREM). If $(\mu, \nu)$ is a quadratic specialization of a quadratic Jordan algebra $\mathfrak{\Im}$ in an associative algebra $\mathfrak{A}$ then

$$
\mu^{\prime}(\alpha 1+x)=\alpha^{2} 1+\alpha \nu(x)+\mu(x)
$$

defines a unital quadratic specialization of the unital quadratic Jordan algebra $\mathfrak{\Im}^{\prime}=\Phi 1+\mathfrak{I}$ in the unital associative algebra $\mathfrak{X}^{\prime}=\Phi 1+\mathfrak{A}$, which extends $(\mu, \nu)$ in the sense that $\mu^{\prime}(x)=\mu(x)$ and $\nu^{\prime}(x)=\nu(x)$ for $x$ in $\Im$.

Proof. Clearly $\mu^{\prime}(x)=\mu(x), \nu^{\prime}(x)=\nu(x)$, so $\mu^{\prime}$ does extend $(\mu, \nu)$. We need to verify

(UCS I) $\mu^{\prime}(1)=1$,

(UCS II) $\mu^{\prime}\left(U_{a}^{\prime} b, a\right)=\mu^{\prime}(a) \nu^{\prime}(b, a)=\nu^{\prime}(a, b) \mu^{\prime}(a)$,

(UCS III) $\mu^{\prime}\left(a^{2}\right)=\mu^{\prime}(a)^{2}$,

(UCS IV) $\mu^{\prime}\left(a^{3}\right)=\mu^{\prime}(a)^{3}$,

for all $a=\alpha 1+x, b=\beta 1+y$ in $\mathfrak{\Im}^{\prime}$. UCS I is obvious. For UCS II, we verify only the first equality:

$$
\begin{aligned}
\mu^{\prime}\left(U_{a}^{\prime} b, a\right)= & \mu^{\prime}\left(\alpha^{2} \beta 1+\alpha^{2} y+2 \alpha \beta x+\alpha x \circ y+\beta x^{2}+U_{x} y, \alpha 1+x\right) \\
= & 2 \alpha^{3} \beta 1+3 \alpha^{2} \beta \nu(x)+\alpha^{3} \nu(y)+\alpha \beta\left\{4 \mu(x)+\nu\left(x^{2}\right)\right\} \\
& +\alpha^{2}\{\mu(y, x)+\nu(x \circ y)\}+\beta \mu\left(x^{2}, x\right)+\alpha\left\{\mu(x \circ y, x)+\nu\left(U_{x} y\right)\right\}+\mu\left(U_{x} y, x\right), \\
\mu^{\prime}(a) \nu^{\prime}(b, a)= & \mu^{\prime}(\alpha 1+x) \nu^{\prime}(\beta 1+y, \alpha 1+x) \\
= & \left\{\alpha^{2} 1+\alpha \nu(x)+\mu(x)\right\}\{2 \alpha \beta 1+\beta \nu(x)+\alpha \nu(y)+\nu(y, x)\} \\
= & 2 \alpha^{3} \beta 1+3 \alpha^{2} \beta \nu(x)+\alpha^{3} \nu(y)+\alpha \beta\left\{2 \mu(x)+\nu(x)^{2}\right\} \\
& +\alpha^{2}\{\nu(y, x)+\nu(x) \nu(y)\}+\beta \mu(x) \nu(x)+\alpha\{\nu(x) \nu(y, x)+\mu(x) \nu(y)\} \\
& \quad+\mu(x) \nu(y, x)
\end{aligned}
$$


which agree by QS I, by linearized QS I and the definition of $\nu(x, y)$, by QS II, by QS III, and by QS IV. Similarly for the second equality in UCS II. For UCS III,

$$
\begin{aligned}
\mu^{\prime}\left(\{\alpha 1+x\}^{2}\right) & =\mu^{\prime}\left(\alpha^{2} 1+2 \alpha x+x^{2}\right) \\
& =\alpha^{4} 1+2 \alpha^{3} \nu(x)+\alpha^{2}\left\{\nu\left(x^{2}\right)+4 \mu(x)\right\}+2 \alpha \mu\left(x^{2}, x\right)+\mu\left(x^{2}\right), \\
\left\{\mu^{\prime}(\alpha 1+x)\right\}^{2} & =\left\{\alpha^{2} 1+\alpha \nu(x)+\mu(x)\right\}^{2} \\
& =\alpha^{4} 1+2 \alpha^{3} \nu(x)+\alpha^{2}\left\{\nu(x)^{2}+2 \mu(x)\right\}+\alpha\{\nu(x) \mu(x)+\mu(x) \nu(x)\}+\mu(x)^{2}
\end{aligned}
$$

which are equal by QS I, QS II, QS V. For UCS IV,

$$
\begin{aligned}
\mu^{\prime}\left(\{\alpha 1+x\}^{3}\right)= & \mu^{\prime}\left(\alpha^{3} 1+3 \alpha^{2} x+3 \alpha x^{2}+x^{3}\right) \\
= & \alpha^{6} 1+3 \alpha^{5} \nu(x)+3 \alpha^{4}\left\{\nu\left(x^{2}\right)+3 \mu(x)\right\}+\alpha^{3}\left\{\nu\left(x^{3}\right)+9 \mu\left(x^{2}, x\right)\right\} \\
& +3 \alpha^{2}\left\{\mu\left(x^{3}, x\right)+3 \mu\left(x^{2}\right)\right\}+3 \alpha \mu\left(x^{3}, x^{2}\right)+\mu\left(x^{3}\right), \\
\left\{\mu^{\prime}(\alpha 1+x)\right\}^{3}= & \left\{\alpha^{2} 1+\alpha \nu(x)+\mu(x)\right\}^{3} \\
= & \alpha^{6} 1+3 \alpha^{5} \nu(x)+3 \alpha^{4}\left\{\mu(x)+\nu(x)^{2}\right\}+3 \alpha^{3}\left\{\nu(x) \mu(x)+\mu(x) \nu(x)+\nu(x)^{3}\right\} \\
& +\alpha^{2}\left\{3 \mu(x)^{2}+\nu(x)^{2} \mu(x)+\nu(x) \mu(x) \nu(x)+\mu(x) \nu(x)^{2}\right\} \\
& +\alpha\left\{\mu(x)^{2} \nu(x)+\mu(x) \nu(x) \mu(x)+\nu(x) \mu(x)^{2}\right\}+\mu(x)^{3}
\end{aligned}
$$

which are equal by QS I, by $\nu\left(x^{3}\right)=\nu(x) \nu\left(x^{2}\right)-\nu(x) \mu(x)=\nu(x)\left\{\nu(x)^{2}-2 \mu(x)\right\}-\nu(x) \mu(x)$ $=\nu(x)^{3}-3 \nu(x) \mu(x)$ (by (19) and QS I-III), by $\mu\left(x^{3}, x\right)=\nu\left(x^{2}\right) \mu(x)=\nu(x)^{2} \mu(x)-2 \mu(x)^{2}$ (QS IV, QS I), by $\mu\left(x^{3}, x^{2}\right)=\nu(x) \mu\left(x^{2}\right)$ (see [5, Lemma 1, (2.15)]), and by QS VI. These relations UCS I-IV hold strictly since $(\mu, \nu)$ remains a quadratic specialization under all scalar extensions. Thus $\mu^{\prime}$ is a unital quadratic specialization.

We call $\mu^{\prime}$ the canonical unital extension of $(\mu, \nu)$.

Given a quadratic representation $(\mu, \nu)$ of $\mathfrak{I}$ on $\mathfrak{M}$ we can form a quadratic algebra, the split null extension $\mathfrak{E}=\mathfrak{I} \oplus \mathfrak{M}$ of $\mathfrak{\Im}$ by $\mathfrak{M}$, by means of the operations

$$
\begin{aligned}
(x \oplus m)^{2} & =x^{2} \oplus \nu_{x}(m), \\
U_{x \oplus m}(y \oplus n) & =U_{x} y \oplus\left\{\mu_{x}(n)+\nu_{x, y}(m)\right\} .
\end{aligned}
$$

Note $\mathfrak{E}$ is a subalgebra of $\mathfrak{F}^{\prime}=\mathfrak{S}^{\prime} \oplus \mathfrak{M}$ since $U_{x \oplus m}^{\prime}(1 \oplus 0)=x^{2} \oplus \nu_{x}(m)=(x \oplus m)^{2}$ and $U_{x \oplus m}^{\prime}(y \oplus n)=U_{x \oplus m}(y \oplus n)$ (note $\nu_{x, y}^{\prime}=\nu_{x}^{\prime} \nu_{y}^{\prime}-\mu_{x, y}^{\prime}=\nu_{x} \nu_{y}-\mu_{x, y}=\nu_{x, y}$ ). Since $\mathfrak{M}$ is a unital $\mathfrak{S}^{\prime}$-bimodule by the Adjunction Theorem, $\mathfrak{F}^{\prime}$ is a unital Jordan algebra by the Unital Bimodule Theorem. Thus the subalgebra $\&$ is also Jordan, and we have

TheOrem 17 (Bimodule TheOREM). The split null extension $\mathfrak{E}=\mathfrak{I} \oplus \mathfrak{M}$ of $a$ quadratic Jordan algebra $\mathfrak{\Im}$ by a bimodule $\mathfrak{M}$ is again a quadratic Jordan algebra.

Once again we see that quadratic specializations, quadratic representations, multiplication representations, and bimodules are all equivalent.

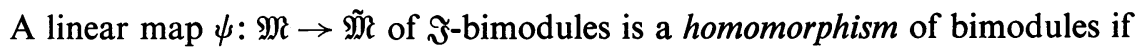

$$
\psi(\nu(x) m)=\tilde{\nu}(x) \psi(m), \quad \psi(\mu(x) m)=\tilde{\mu}(x) \psi(m) .
$$

If $\mathfrak{M}, \tilde{\mathfrak{M}}$ are unital $\mathfrak{\Im}$-bimodules this reduces to the previous definition since $\nu(x)=\mu(x, 1), \tilde{\nu}(x)=\tilde{\mu}(x, 1)$. 
The kernel of a quadratic specialization $(\mu, \nu)$ is

$$
\operatorname{Ker}(\mu, \nu)=\{z \mid \mu(z)=\nu(z)=\mu(z, x)=0 \text { for all } x\} .
$$

In the case of unital specializations this coincides with the earlier definition. Indeed, $\operatorname{Ker}(\mu, \nu)$ is just the intersection $\Im \cap \operatorname{Ker} \mu^{\prime}$ of $\Im$ with the kernel of the canonical unital extension $\mu^{\prime}:$ if $z \in \operatorname{Ker}(\mu, \nu)$ then $\mu^{\prime}(z)=\mu(z)=0, \mu^{\prime}\left(z, x^{\prime}\right)=$ $\mu^{\prime}(z, \alpha 1+x)=\alpha \nu(z)+\mu(z, x)=0$, while if $z \in \Im \cap \operatorname{Ker} \mu^{\prime}$ then $\nu(z)=\mu^{\prime}(z, 1)=0$, $\mu(z)=\mu^{\prime}(z)=0, \mu(z, x)=\mu^{\prime}(z, x)=0$. In particular, we conclude $\operatorname{Ker}(\mu, \nu)$ is an ideal in $\Im$. The induced specialization $(\bar{\mu}, \bar{\nu})$ of $\bar{\Im}=\Im / \operatorname{Ker}(\mu, \nu)$ given by $\bar{\mu}(x+K)$ $=\mu(x), \bar{\nu}(x+K)=\nu(x)$ is faithful in the sense that $\operatorname{Ker}(\bar{\mu}, \bar{\nu})=0$.

If the kernel is all of $\Im$, so $\mu \equiv \nu \equiv 0$, we say $(\mu, \nu)$ is a zero quadratic specialization; similarly we have zero representations and zero bimodules.

We could follow the same procedure as in the unital case to define cohomology groups $H^{\prime n}(\Im, \mathfrak{M})$ in the category of quadratic Jordan algebras, but it would turn out that the nonunital cohomology coincided with the unital cohomology of the canonical unital extension $\mathfrak{\Im}^{\prime}$ :

$$
H^{\prime n}(\mathfrak{J}, \mathfrak{M})=H^{n}\left(\mathfrak{I}^{\prime}, \mathfrak{M}\right)
$$

2. Universal gadgets. A universal quadratic envelope for $\Im$ consists of an associative algebra $\mathscr{2} \mathscr{E}(\Im)$ together with a universal quadratic specialization $(u, v): \Im \rightarrow \mathscr{2} \mathscr{E}(\Im)$ such that any quadratic specialization $(\mu, \nu)$ factors uniquely through $(u, v)$ :

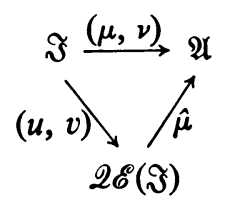

$\mathscr{2} \mathscr{E}(\Im)$ exists and satisfies the usual properties (but note that $\mathscr{2} \mathscr{E}(\Im)$ is not assumed to be unital). One way to establish the basic properties is to observe

Proposition 18. If $\Im$ is a quadratic Jordan algebra and $\mathfrak{J}^{\prime}=\Phi 1+\Im$ the canonical unital extension of $\Im$ then the subalgebra $\mathscr{Q} \mathscr{E}(\Im)$ of $\mathscr{U} \mathscr{Q} \mathscr{E}\left(\Im^{\prime}\right)$ generated by $u^{\prime}(\Im), v^{\prime}(\Im)$ together with the restrictions $u$ and $v$ of the universal unital quadratic specialization $u^{\prime}$ and $v^{\prime}$ to $\Im$ forms a universal quadratic envelope for $\Im$.

Proof. Contemplate the diagram

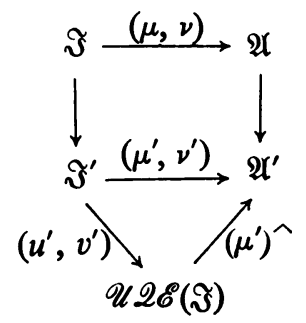


using the Adjunction Theorem; observe that the restriction $\hat{\mu}$ of $\left(\mu^{\prime}\right)^{\wedge}$ to $\mathscr{U} \mathscr{Q}(\Im)$ has its range in $\mathfrak{A}$ since this is true for the generators $\left(\mu^{\prime}\right)^{\wedge}\left(u^{\prime}(\mathfrak{I})\right)=\mu^{\prime}(\mathfrak{I})=\mu(\mathfrak{\Im})$, $\left(\mu^{\prime}\right)^{\wedge}\left(v^{\prime}(\Im)\right)=\nu^{\prime}(\Im)=\nu(\Im)$.

What happens if $\mathfrak{I}$ is already unital? Suppose that $\mathfrak{I}$ is a quadratic Jordan algebra with unit 1 . We wish to compare its universal quadratic envelopes in the category of unital quadratic and (plain old) quadratic Jordan algebras.

THEOREM 19. If $\mathfrak{I}$ is a quadratic Jordan algebra with unit then the universal quadratic envelope has

$$
\mathscr{Q} \mathscr{E}(\Im)=\mathfrak{Q}_{1} \oplus \mathfrak{Q}_{0}, \quad u=u_{1} \oplus u_{0}, \quad v=v_{1} \oplus v_{0}
$$

for ideals $\mathfrak{Q}_{i}$ where $\left(\mathfrak{Q}_{1}, u_{1}\right)$ serves as a universal unital quadratic envelope for $\Im$ and

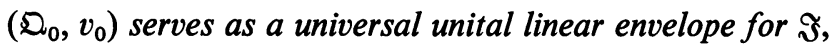

$$
\mathscr{2} \mathscr{E}(\Im)=\mathscr{U} \mathscr{Q} \mathscr{E}(\Im) \oplus \mathscr{U} \mathscr{L} \mathscr{E}(\Im)
$$

and where $v_{1}(x)=u_{1}(x, 1), u_{0}(x) \equiv 0$.

Proof. It is not too difficult to verify this directly, but we prefer to reduce it to the unital case.

The canonical unital extension $\mathfrak{\Im}^{\prime}=\Phi 1+\mathfrak{T}$ splits into a direct sum $\mathfrak{F}^{\prime}=\mathfrak{I}_{1} \oplus \mathfrak{\Im}_{2}$ of ideals $\Im_{1}=\Im, \Im_{2}=\Phi e_{2}$ where $e_{2}=1-e_{1}, e_{1}$ the unit for $\Im$. Thus we have seen in Proposition 13 that $\mathscr{U} \mathscr{Q} \mathscr{E}\left(\Im^{\prime}\right)=\mathfrak{Q}_{1} \oplus \mathfrak{Q}_{0} \oplus \mathfrak{Q}_{2}, u^{\prime}=u_{1}^{\prime} \oplus u_{0}^{\prime} \oplus u_{2}^{\prime}$, for $\left(\mathfrak{Q}_{1}, u_{1}^{\prime}\right)$ the universal unital envelope of $\mathfrak{I}_{1}$ and $\left(\mathfrak{Q}_{0}, u_{0}^{\prime}\right)$ the universal unital compound linear envelope of $\left(\Im_{1}, \Im_{2}\right)$. By Theorem $18, \mathscr{Q} \mathscr{E}(\Im)$ is the subalgebra of $\mathscr{U} \mathscr{Q} \mathscr{E}\left(\Im^{\prime}\right)$ generated by $u^{\prime}(\Im)=u_{1}^{\prime}\left(\Im_{1}\right)$ and $v^{\prime}(\Im)=u^{\prime}\left(\Im_{1}, e_{1}+e_{2}\right)=v_{1}^{\prime}\left(\Im_{1}\right)+v_{0}^{\prime}\left(\Im_{1}\right)$. This subalgebra is just $\mathfrak{Q}_{1} \oplus \mathfrak{Q}_{0}$ since $u_{1}^{\prime}\left(\mathfrak{\Im}_{1}\right)$ generates $\mathfrak{Q}_{1}$ and $v_{0}^{\prime}\left(\mathfrak{\Im}_{1}\right)=u^{\prime}\left(\Im_{1}, e_{2}\right)=u^{\prime}\left(\Im_{1}, \Phi e_{2}\right)=u^{\prime}\left(\Im_{1}, \mathfrak{I}_{2}\right)$ generates $\mathfrak{Q}_{0}$. This immediately gives $\left(\mathfrak{Q}_{1}, u_{1}^{\prime}\right)=\left(\mathscr{U} \mathscr{Q} \mathscr{E}\left(\mathfrak{\Im}_{1}\right), u_{1}\right)$. Also, since $\Im_{2}=\Phi e_{2}$ it is easy to verify $\mathscr{U} \mathscr{L} \mathscr{E}\left(\Im_{2}\right)=\Phi$, so $\mathscr{U} \mathscr{C} \mathscr{E}\left(\Im_{1}, \Im_{2}\right)=\mathscr{U} \mathscr{L} \mathscr{E}\left(\Im_{1}\right) \otimes \mathscr{U} \mathscr{L} \mathscr{E}\left(\Im_{2}\right)$ $=\mathscr{U} \mathscr{L} \mathscr{E}(\Im)$ and $c=l_{1} \otimes l_{2}=l$ (the universal unital linear specialization of $\left.\Im\right)$. Thus $\left(\mathfrak{Q}_{0}, v_{0}^{\prime}\right)=(\mathscr{U} \mathscr{L} \mathscr{E}(\Im), l)$ is the universal unital linear envelope (note that $u_{0}^{\prime}(\Im) \equiv 0$ but $v_{0}^{\prime}(x)=u_{0}^{\prime}\left(x_{1}, e_{2}\right)=c(x)=l(x)$ for $x=x_{1}$ in $\left.\Im\right)$.

The above theorem suggests that a unital Jordan algebra should have some nonunital quadratic specializations which come from unital linear specializations (these would account for the summand $\mathfrak{Q}_{0}$ in $\mathscr{Q} \mathscr{E}(\mathfrak{\Im})$ ). Indeed, just as in the unital case,

Proposition 20. If $\sigma, \tau$ are commuting linear specializations of a quadratic Jordan algebra $\mathfrak{\Im}$ in $\mathfrak{A}$ then $\mu=\sigma \tau, \nu=\sigma+\tau$ defines a quadratic specialization of $\mathfrak{\Im}$ in $\mathfrak{A}$.

Proof. $\sigma, \tau$ extend to unital specializations $\sigma^{\prime}, \tau^{\prime}$ of $\mathfrak{\Im}^{\prime}$ in $\mathfrak{A}^{\prime}$ for $\mathfrak{J}^{\prime}=\Phi 1+\mathfrak{\Im}$, $\mathfrak{A}^{\prime}=\Phi 1+\mathfrak{A}$, so by the unital result $\mu^{\prime}=\sigma^{\prime} \tau^{\prime}$ is a unital quadratic specialization with $\nu^{\prime}=\sigma^{\prime}+\tau^{\prime}$ since $\nu^{\prime}(x)=\mu^{\prime}(x, 1)=\sigma^{\prime}(x) \tau^{\prime}(1)+\sigma^{\prime}(1) \tau^{\prime}(x)=\sigma^{\prime}(x)+\tau^{\prime}(x)$. By restriction $\mu=\sigma \tau, \nu=\sigma+\tau$ affords a quadratic specialization of $\mathfrak{\Im}$ in $\mathfrak{A}$. 
Again we call these compound linear specializations. Our calculations show $(\mu, \nu)$ is compound linear if and only if the canonical unital extension $\mu^{\prime}$ is compound linear.

In particular, any linear specialization $\sigma$ of $\Im$ commutes with $\tau=0$, so $\sigma$ determines a quadratic specialization with $\mu \equiv 0, \nu \equiv \sigma$. Conversely, any quadratic specialization with $\mu \equiv 0$ has $\sigma=\nu$ a linear specialization: we have $\sigma\left(x^{2}\right)=\sigma(x)^{2}$ and $\sigma\left(U_{x} y\right)$ $=\sigma(x) \sigma(y) \sigma(x)$ since $\nu(x)^{2}-\nu\left(x^{2}\right)=2 \mu(x)=0$ and $\nu\left(U_{x} y\right)=\nu(x) \nu(y, x)-\nu(y) \mu(x)$ $=\nu(x) \nu(y) \nu(x)$ if $\mu(x)=\mu(x, y)=0$ (see QS I' and (19)). Note that the corresponding representation $\rho=\frac{1}{2} \nu=\frac{1}{2} \sigma$ for the linear Jordan algebra again requires $\frac{1}{2} \in \Phi$.

Proposition 21. The quadratic specializations $(\mu, \nu)$ of $\Im$ with $\mu \equiv 0$ are precisely the compound linear specializations $\mu=\sigma \tau, \nu=\sigma+\tau$ determined by a linear specialization $\sigma$ and $\tau=0$.

From Theorem 19 we get

COROllaRY 22. Any quadratic specialization $(\mu, \nu)$ of a quadratic Jordan algebra $\Im$ with unit is a direct sum $\mu=\mu_{1} \oplus \mu_{0}, \nu=\nu_{1} \oplus \nu_{0}$ for $\mu_{1}$ a unital quadratic specialization, $\nu_{0}$ a unital linear specialization, and $\mu_{0} \equiv 0, \nu_{1}(x) \equiv \mu_{1}(x, 1)$.

If $\sigma$ is a linear specialization of $\mathfrak{I}$ in $\mathfrak{A}=\operatorname{Hom}_{\Phi}(\mathfrak{M}, \mathfrak{M})$ we call it a linear representation of $\mathfrak{J}$ on $\mathfrak{M}$, and call $(\mathfrak{M}, \sigma)$ a module for $\mathfrak{\Im}$. Similarly we can speak of unital linear representations and unital modules. If $\mathfrak{F}=\mathfrak{A}^{+}$for $\mathfrak{A}$ associative then left or right $\mathfrak{U}$-modules $\mathfrak{M}$ (i.e. homomorphisms or antihomomorphisms of $\mathfrak{A}$ in $\operatorname{Hom}_{\Phi}(\mathfrak{M}, \mathfrak{M})$ ) are $\mathfrak{\Im}$-modules. Thus Jordan modules are sort of an analogue of one-sided associative modules; note, however, that exceptional Jordan algebras cannot have any faithful modules at all.

COROLlaRY 23. Any bimodule $\mathfrak{M}$ for a quadratic Jordan algebra $\mathfrak{\Im}$ with unit can be decomposed into a direct sum

$$
\mathfrak{M}=\mathfrak{M}_{1} \oplus \mathfrak{M}_{1 / 2} \oplus \mathfrak{M}_{0}
$$

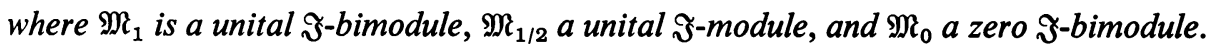

We again say a bimodule $\mathfrak{M}$ for $\mathfrak{\Im}$ is special if the split null extension $\mathfrak{E}=\mathfrak{I} \oplus \mathfrak{M}$ is a special quadratic Jordan algebra. $\mathfrak{M}$ is special for $\mathfrak{\Im}$ if and only if it is special for $\mathfrak{\Im}^{\prime}$, so from the unital case we deduce

Proposition 24. A bimodule $(\mathfrak{M}, \mu, \nu)$ for a quadratic Jordan algebra $\Im$ is special if and only if the representation $(\mu, \nu)$ is a compound linear representation.

An algebra is strongly special if all its bimodules are special. Since any zero bimodule is trivially special, and any module is special since its quadratic representation is compound linear, an algebra with unit is strongly special in the category of all quadratic Jordan algebras if and only if it is strongly special in the category of unital quadratic Jordan algebras. 
3. The finiteness theorem. Now we prove the important result that if $\mathfrak{\Im}$ is finitely spanned then so is its universal quadratic envelope. We shall not attempt to get a very precise bound on the dimension of $\mathscr{Q} \mathscr{E}(\Im)$.

THEOREM 25 (FINITENESS THEOREM). If the quadratic Jordan algebra $\Im$ is spanned by elements $x_{1}, \ldots, x_{n}$ then the universal quadratic envelope $\mathscr{Q} \mathscr{E}(\Im)$ is spanned by elements

$$
u\left(y_{i_{1}}\right) \cdots u\left(y_{i_{r}}\right) v\left(x_{j_{1}}\right) \cdots v\left(x_{j_{s}}\right)
$$

for $i_{1}<i_{2}<\cdots<i_{r}, j_{1}<j_{2}<\cdots<j_{s}(0 \leqq r \leqq n(n+1) / 2,0 \leqq s \leqq n)$ where the $y_{i}$ are the $n(n+1) / 2$ elements $x_{i i}=x_{i}$ and $x_{i j}=x_{i}+x_{j}=x_{j i}(i \neq j)$ in some order. In particular, $\mathscr{Q} \mathscr{E}(\Im)$ is finitely spanned.

Proof. A spanning set is certainly a generating set, so by the Multiplication Closure Theorem we know $\mathscr{2} \mathscr{E}(\Im)$ is generated by the $v\left(x_{i}\right), u\left(x_{i}\right)$, and $u\left(x_{i}, x_{j}\right)$. Since $u\left(x_{i}\right)=u\left(x_{i i}\right)$ and $u\left(x_{i}, x_{j}\right)=u\left(x_{i}+x_{j}\right)-u\left(x_{i}\right)-u\left(x_{j}\right)=u\left(x_{i j}\right)-u\left(x_{i i}\right)-u\left(x_{j j}\right)$ we see that the $v\left(x_{i}\right)$ and $u\left(y_{i}\right)$ generate too. Thus we dispense with any $u(x, y)$ 's.

The heart of the proof is the commutativity formulas

(i) $v(x)^{2}=v\left(x^{2}\right)+2 u(x)$,

(ii) $v(x) v(y)+v(y) v(x)=v(x \circ y)+2 u(x, y)$,

(iii) $v(x) u(y)+u(y) v(x)=u(x \circ y, y)$,

(iv) $u(x)^{2}=u\left(x^{2}\right)$,

(v) $u(x) u(y)-u(y) u(x)=u\left(y, U_{x} y\right)+u(x \circ y)-u(x \circ y, y) v(x)+u(y) v\left(x^{2}\right)$.

Most of these we have seen before: (i) is QS I' and (ii) its linearization; (iii) is just (20); (iv) is QS V. For (v), we have

$$
\begin{aligned}
{[u(x), u(y)] } & =u(x) u(y)+u(y) u(x)-2 u(y) u(x) \\
& =-v(x) u(y) v(x)+u\left(y, U_{x} y\right)+u(x \circ y)-u(y)\left\{v(x)^{2}-v\left(x^{2}\right)\right\}
\end{aligned}
$$

(by QS I and by linearizing $x \rightarrow x, 1$ in QS VI in the unital extension of $u$ )

$$
\begin{aligned}
& =-\{v(x) u(y)+u(y) v(x)\} v(x)+u\left(y, U_{x} y\right)+u(x \circ y)+u(y) v\left(x^{2}\right) \\
& =-u(x \circ y, y) v(x)+u\left(y, U_{x} y\right)+u(x \circ y)+u(y) v\left(x^{2}\right) .
\end{aligned}
$$

From these we can use a "straightening" argument to put the monomials spanning $2 \mathscr{E}(\Im)$ in the "normal form" of the theorem. To each monomial consisting of $u(y)$ 's and $v(x)$ 's we associate a total degree $n$ (the number of $u$ 's and $v$ 's) and a $u$-degree $m$ (the number of $u$ 's). We proceed to straighten monomials by a double induction on $n$ and $m$, assuming any monomial of total degree $<n$ or total degree $n$ but $u$-degree $<m$ has already been straightened (for $n=m=0$ the trivial monomial $I$ is already straight enough). Then it suffices to prove any monomial of total degree $n$ and $u$-degree $m$ is congruent to a straightened monomial modulo monomials of lower degree.

The first step is to move all the $v$ 's to the right by means of (iii), then to arrange $v\left(x_{j_{1}}\right) \cdots v\left(x_{j_{r}}\right)$ so that $j_{1} \leqq j_{2} \leqq \cdots \leqq j_{r}$ by the commutativity relation (ii), and then so 
that $j_{1}<j_{2}<\cdots<j_{r}$ by dropping repeated terms using (i). It remains to straighten a product of $u(y)$ 's. We use a similar process here, getting $u\left(y_{i_{1}}\right) \cdots u\left(y_{i_{s}}\right)$ with $i_{1} \leqq i_{2} \leqq \cdots \leqq i_{s}$ from (v) (here for the first time the right side of (v) does not consist entirely of terms of lower total degree, but those terms that still have total degree 2 all have $u$-degree 1 instead of 2), and dropping repeated terms so $i_{1}<i_{2}<\cdots<i_{s}$ by (iv).

Note that the estimate for the dimension of $\mathscr{2} \mathscr{E}(\mathfrak{J})$ is

$$
\sum_{r=0}^{N}\left(\begin{array}{l}
N \\
r
\end{array}\right) \sum_{s=0}^{n}\left(\begin{array}{l}
n \\
s
\end{array}\right)=2^{N} 2^{n}=2^{n(n+3) / 2}, \quad N=n+\frac{n(n-1)}{2}=\frac{n(n+1)}{2},
$$

whereas the estimate in the case of linear Jordan algebras is only $\left(\begin{array}{c}2 n+1 \\ n\end{array}\right)$ [3, p. 97]. Of course, because of the presence of "pathological" representations we expect $\mathscr{2} \mathscr{E}(\Im)$ to be bigger than in the linear case.

Also note that since $\mathscr{U} \mathscr{Q} \mathscr{E}(\mathfrak{I})$ is contained in $\mathscr{2} \mathscr{E}(\mathfrak{I})$ if $\mathfrak{\Im}$ is unital, $\mathscr{U} \mathscr{Q} \mathscr{E}(\mathfrak{I})$ is finite dimensional if the unital algebra $\mathfrak{\Im}$ is.

\section{REFERENCES}

1. P. Cohn, Universal algebra, Harper and Row, New York, 1965. MR 31 \#224.

2. S. Eilenberg, Extensions of general algebras, Ann. Soc. Polon. Math. 21 (1948), 125-134. MR 10, 180.

3. N. Jacobson, Structure and representations of Jordan algebras, Amer. Math. Soc. Colloq. Publ., vol. 39, Amer. Math. Soc., Providence, R. I., 1968.

4. K. McCrimmon, A general theory of Jordan rings, Proc. Nat. Acad. Sci. U. S. A. 56 (1966), 1072-1079. MR 34 \#2643.

5. - Quadratic Jordan algebras and cubing operations, Trans. Amer. Math. Soc. 153 (1971), 265-278.

6. - Jordan algebras with interconnected idempotents, Proc. Amer. Math. Soc. 19 (1968), 1327-1336. MR 38 \#203.

UNIVERSITY OF VIRGINIA,

Charlottesville, Virginia 22901 